Auditory Superiority for Perceiving the Beat Level but not Measure Level in Music Jessica E. Nave-Blodgett ${ }^{1,2}$, Joel S. Snyder ${ }^{1}$, and Erin E. Hannon ${ }^{1}$

${ }^{1}$ Department of Psychology, University of Nevada, Las Vegas

${ }^{2}$ Exponent Inc., 23445 North 19th Ave, Phoenix, AZ 85027, USA

Word Count: 16,205

Revision submitted to Journal of Experimental Psychology: Human Perception and

Performance,

$06 / 03 / 2021$

Preprint posted on PsyArXiv on 06/04/2021 (pre-peer review)

Draft version 2.00, 06/04/2021. This paper has not been peer reviewed. Please do not copy or cite without authors' permission.

Author Note:

Jessica E. Nave-Blodgett, Department of Psychology, University of Nevada, Las Vegas and Exponent Inc., 23445 North 19th Ave, Phoenix, AZ 85027, USA; Joel S. Snyder, Department of Psychology, University of Nevada, Las Vegas; Erin E. Hannon, Department of Psychology, University of Nevada, Las Vegas.

Research and manuscript preparation supported in part by a University of Nevada Las Vegas Foundation Board of Trustees Fellowship and a Barrick Graduate Fellowship awarded to Jessica E. Nave-Blodgett.

Portions of the results from this manuscript have previously been presented at the following conferences: the Mid-Winter Meeting of the Association for Research in 
Otolaryngology (February 2016), the New England Sequencing and Timing Forum (March 2016), the International Conference on Music Perception and Cognition (August 2016), and the joint meeting of the Acoustical Society of America and the Acoustical Society of Japan (November 2016). Experiment 2 of this manuscript has previously been reported as part of an unpublished thesis for the completion of a Masters of Arts in Psychology by the first author. Correspondence concerning this article should be addressed to Jessica Nave-Blodgett, Exponent Inc., 23445 North 19th Ave, Phoenix, AZ 85027, USA.

Email: jes.nave@gmail.com 


\begin{abstract}
Auditory perception of time is superior to visual perception, both for simple intervals and beatbased musical rhythms. To what extent does this auditory advantage characterize perception of different hierarchical levels of musical meter, and how is it related to lifelong experience with music? We paired musical excerpts with auditory and visual metronomes that matched or mismatched the musical meter at the beat level (faster) and measure level (slower) and obtained fit ratings from adults and children (5-10 years). Adults exhibited an auditory advantage in this task for the beat level, but not for the measure level. Children also displayed an auditory advantage that increased with age for the beat level. In both modalities, their overall sensitivity to beat increased with age, but they were not sensitive to measure-level matching at any age. More musical training was related to enhanced sensitivity in both auditory and visual modalities for measure-level matching in adults and beat-level matching in children. These findings provide evidence for auditory superiority of beat perception across development, and they suggest that beat and meter perception develop quite gradually and rely on lifelong acquisition of musical knowledge.
\end{abstract}

Keywords: time perception, beat perception, meter perception, modality differences, development

Public Significance Statement: This study examines how perception of musical rhythms is better for auditory than for visual patterns in children and young adults, and whether this is affected by extensive music and dance experience. The findings could be helpful for providing music education tailored for particular age groups, and for justifying the importance of music education in facilitating the development of auditory and visual temporal processing. 


\section{Auditory Superiority for Perceiving the Beat Level but not Measure Level in Music}

Music and speech are universal human behaviors that rely on the ability to perceive and produce temporal patterns. Both music and speech contain periodic information nested at multiple timescales ranging from the tens of milliseconds to periods longer than a second (Ding et al., 2017; Kello et al., 2017). Our perception of these hierarchically-nested periodicities may underpin our ability to coordinate our behavior with music alone or with small and large groups, and may increase intelligibility in speech (Beier \& Ferreira, 2018; Ding et al., 2016; Riecke et al., 2018). Many studies have demonstrated an advantage for the auditory system over the visual system for the perception and production of simple temporal intervals as well as complex rhythms with regular, periodic organization. However, it is unclear if this auditory advantage extends to the perception of slower levels of temporal structure, such as the multiple, hierarchically-related beat periods of musical meter. Furthermore, it is unknown if this auditory advantage for rhythm perception arises early in development or depends on the accumulation of lifelong experience with predominantly auditory rhythms in music and speech. We conducted several experiments to better understand the developmental origins, characteristics, and mechanisms of human rhythm perception in vision and audition.

In music, beat refers to the perceptual pattern of quasi-isochronous periodic pulsations in time to which one would move while listening to music (Lerdahl \& Jackendoff, 1985; Lester, 1986). Listeners have a strong tendency to perceive a salient beat with an inter-onset interval that ranges from 300-900 $\mathrm{ms}$ in rhythms as simple as discrete isochronous events and as complex as continuous, overlapping multi-instrument music with no discrete onsets or offsets of sound (Drake, Jones, et al., 2000; Fraisse, 1982; Handel \& Oshinsky, 1981; Parncutt, 1994; van Noorden \& Moelants, 1999). While the primary beat is perceptually salient, according to music 
theorists, it is only one hierarchical level of musical meter, which comprises multiple levels of periodic structure that multiply or subdivide the period of the beat (Lehrdahl \& Jackendoff, 1985; London, 2002, 2012). Beat and meter are psychological phenomena which arise from the interaction of an individual's accumulated experience engaging with the music of their culture, and the physical properties of the auditory input (London et al., 2017; van der Weij et al., 2017). Adults use multiple sources of information to infer the location of beats in music, including occurrences of louder events, longer events, salient melodic features, and events that continue previously established patterns (Desain \& Honing, 2003; Hannon et al., 2004; Jones \& Pfordresher, 1997; Palmer \& Krumhansl, 1990). When two or more of these levels align, individuals subjectively experience that beat as stronger or more salient than other beats (Figure 1). Meter perception, by definition, requires an individual to perceive multiple hierarchical levels of beat simultaneously.

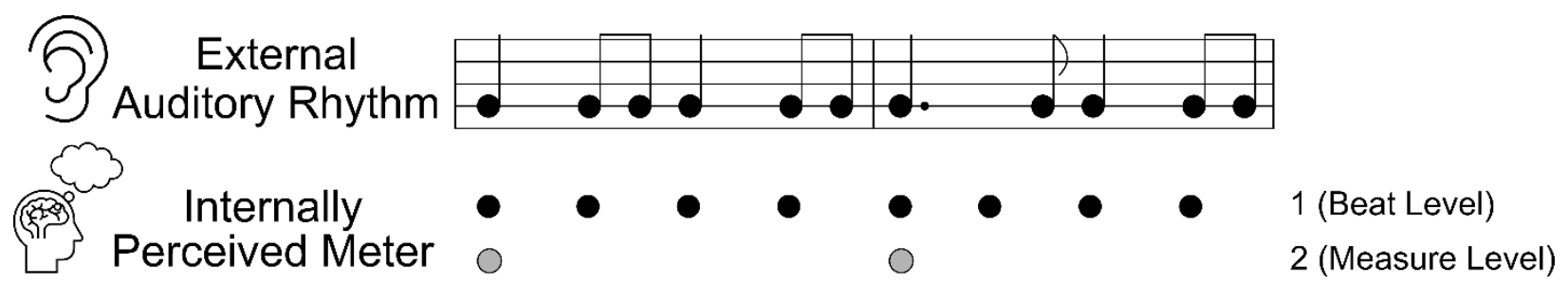

Figure 1. An illustration of the surface-level temporal structure of a western-music rhythm (musical notes) and two possible perceived levels of beat supported by the rhythm (circles), which make up two levels of the meter (beat and measure). Points in time when multiple metrical levels align, such as the downbeat of the measure, tend to be perceived as stronger. The perception of meter is heavily influenced by experience, often by the stylistic norms of the musical culture an individual is most familiar with.

Beat perception can arise from auditory, visual, or somatosensory stimulation (Huang et al., 2012; Nave-Blodgett et al., 2021; C. Palmer \& Krumhansl, 1990; S. Palmer \& Peterson, 
2016; $\mathrm{Su}, 2016 \mathrm{a}$ ), suggesting that beat (and possibly meter) perception is not exclusive to the auditory modality. There is evidence that beat perception relies on either shared or partially overlapping auditory and visual processes (Grahn et al., 2011; Marchant \& Driver, 2013; Schubotz et al., 2000). Adults can tap to the beat of both auditory (Drake, Penel, et al., 2000; Parncutt, 1994; van Noorden \& Moelants, 1999; for a review, see Repp \& Su, 2013) and visual sequences (Su, 2016a, 2016b). Adults also show a beat-based advantage, whereby they more easily reproduce and discriminate rhythms that induce a strong percept of a beat over those that do not (or only weakly) induce a beat percept, for both auditory (Essens \& Povel, 1985; Grahn, 2012; Grahn \& Brett, 2007; Grahn \& Rowe, 2009; Povel, 1981; Povel \& Essens, 1985) and visual rhythms (Grahn, 2012). Similarly, adults can judge if a probe event falls on or off a beat in both auditory (C. Palmer \& Krumhansl, 1990) and visual contexts (S. Palmer \& Peterson, 2016). Some evidence nevertheless suggests there may be specialized mechanisms in auditory pathways that are more optimal for temporal processing. For example, the auditory system consistently outperforms the visual system in the perception of short, isolated temporal intervals and of more complex rhythmic patterns (Grondin, 1993, 2010; van Wassenhove, 2009). Even when presented with the same rhythms in visual and auditory modalities, adults are worse at detecting the beat of the visual version (Grahn et al., 2011; McAuley \& Henry, 2010; Pasinski et al., 2016), and they are worse at discriminating between pairs of visual rhythms than between pairs of auditory rhythms (Grahn, 2012). Individuals are also more accurate at reproducing auditory versus visual versions of otherwise identical rhythms (Gault \& Goodfellow, 1938; Huff, 1972; Kosonen \& Raisamo, 2006). Adults exhibit higher variability and lower accuracy when tapping to visual than to auditory rhythms (Iversen et al., 2015; Patel et al., 2005; Repp, 2003; for a review, see Repp \& Su, 2013), and they have a harder time ignoring auditory than visual 
distractors when tapping to rhythms in either modality (Repp \& Penel, 2002, 2004). This auditory advantage for beat synchronization decreases - but does not completely disappear when spatial movement is added to visual stimuli (Hove et al., 2010; Hove, Fairhurst, et al., 2013; Hove, Iversen, et al., 2013; Mu, et al., 2018; Su, 2014a, 2014b; Su \& Salazar-López, 2016), suggesting the auditory advantage may diminish when visual stimuli have greater complexity and ecological validity.

It is also possible that the observed auditory advantage for beat perception arises largely from the accumulation of lifelong experience with predominantly auditory rhythms. Musicians, who might have more extensive experience with musical rhythms than a typical listener, demonstrate enhanced auditory beat perception and synchronization skills compared to nonmusicians (Drake, 1993; Drake, Penel, et al., 2000; Geiser et al., 2010; Grahn \& Rowe, 2009; Grahn \& Schuit, 2012; Karpati et al., 2016; Manning \& Schutz, 2016; Matthews et al., 2016; Nave-Blodgett et al., 2021; Palmer \& Krumhansl, 1990; Skaansar et al., 2019). Conversely, congenitally deaf individuals who rely on visual temporal acuity for communication purposes are as accurate at tapping with visual-only metronomes as hearing individuals are with auditory metronomes (Iversen et al., 2015). This suggests the amount of experience engaging with rhythmic structures in the auditory or visual modalities, rather than inherently superior auditory temporal processing, may drive how well individuals perceive and produce auditory versus visual rhythms. Because music is usually experienced by most individuals through sound, even adults with no formal training may demonstrate an auditory advantage for beat perception - and possibly meter perception - due to greater experience with auditory rhythms in speech and music compared to visual rhythms. 
In principle, music training might lead to enhancement of beat and meter perception across modalities, rather than just in the auditory modality, especially if meter perception is driven by an amodal process of directing attention in time (Jones, 1976; Jones \& Boltz, 1989; Large \& Jones, 1999). Domain-general attention does seem to strongly contribute to the ability to perceive beat and meter (Celma-Miralles \& Toro, 2019; Chapin et al., 2010). Increased musical training predicts greater attention to slower levels of meter (Nave-Blodgett et al., 2021; Palmer \& Krumhansl, 1990), and this is reflected in musicians' stronger neural responses to metricallystructured sounds (Celma-Miralles et al., 2016; Celma-Miralles \& Toro, 2019; Geiser et al., 2010). Musicians demonstrate superior temporal perception in both audition and vision (Rammsayer \& Altenmüller, 2006; Rammsayer et al., 2012), and superior rhythm discrimination for both visual and auditory rhythms compared to non-musicians (Grahn, 2012). Musicians' audiovisual temporal binding window is also narrower and more precisely centered than nonmusicians' (Bidelman, 2016), suggesting there is an association between music training and multisensory perception.

Like musicians, dancers also show enhanced perception in both auditory and visual modalities. Given only visual information, trained dancers are better than non-dancers at synchronizing their movements with a novel dance partner (Washburn et al., 2014), discriminating between point-light figures dancing with different movements (Calvo-Merino et al., 2010), and at sensorimotor synchronization to auditory stimuli (Huff, 1972; Jin et al., 2019; Miura et al., 2016). Experienced dancers also articulate more levels of metrical timing in their movement than less-experienced dancers (Naveda et al., 2015, Naveda \& Lehman, 2010), suggesting possible greater awareness and thus embodiment of metrical structure. When directly compared with musicians, dancers outperform musicians on whole-body coordination tasks, but 
musicians outperform dancers on auditory rhythm synchronization tasks (Karpati et al., 2016), suggesting that dance training and music training relate to perception and production tasks in different ways. Of course, it is also possible that those with training may have had pre-existing temporal processing advantages compared to those individuals who did not pursue music or dance training.

One way of investigating how experience influences modality advantages for beat and meter processing is to examine younger individuals who are still accumulating experience. If the auditory advantage for beat perception is driven primarily by experience, then we might expect the auditory advantage for beat perception to increase with age. By contrast, if the auditory advantage is mostly the result of intrinsically superior timing in the auditory modality, we might expect an auditory advantage to be present early in childhood and remain relatively stable throughout development. If the auditory advantage begins with intrinsically superior auditory timing but is enhanced by accumulated experience, we might expect the auditory advantage to be present early on and increase with age.

There is some evidence that robust auditory advantages for temporal processing exist early in development: rhythm perception in vision lags behind audition during development. However, auditory experience also precedes visual experience, since the auditory system is functioning and receiving external input by 25 weeks into gestation, whereas the visual system receives minimal external sensory input before birth (Cheour-Luhtanen et al., 1996). With auditory rhythms, infants as young as 2 months can discriminate between different rhythmic patterns (Demany et al., 1977), and changes in tempo (Baruch \& Drake, 1997; Thorpe \& Trehub, 1989). However, with visual rhythms, it is not until 5 months of age that infants detect tempo changes (Bahrick et al., 2002), and not until 7 months that infants discriminate between different 
visual rhythms (Bahrick \& Lickliter, 2004). Even then, rhythm discrimination is only observed if the visual rhythms contain apparent motion cues (Brandon \& Saffran, 2011). Children's synchronization to auditory and visual metronomes and rhythms improves gradually with increasing age (Braun Janzen, et al., 2014; Drake, 1993; Drake, Jones, et al., 2000; McAuley et al., 2006; Mu et al., 2018; Provasi \& Bobin-Bègue, 2003; Thompson et al., 2015), but they remain less accurate and more variable with visual than auditory metronomes and rhythms until later in adolescence (Kurgansky \& Shupikova, 2011; Mu et al., 2018; Rosenbusch \& Gardner, 1968).

Even though auditory temporal perception outperforms visual temporal perception during childhood, there is nevertheless a shift with age from relying on auditory to visual information when making temporal judgments. Presented with simultaneous sounds and images, young children are more likely to report they heard a sound (but did not see an image) while older children report they saw an image rather than hearing a sound, suggesting a shift from auditory to visual dominance in an audiovisual conflict task (Nava \& Pavani, 2013). Similarly, when asked to report how many flashes they saw or beeps they heard, younger children under- or overreport flashes to match the number of beeps, while older children and adults tend to over- or under-report the number of beeps to match the number of flashes (Innes-Brown et al., 2011; Nava \& Pavani, 2013; Parker \& Robinson, 2018). Children's cross-modal temporal binding window is also larger than adults: in simultaneity judgment tasks, when an auditory stimulus leads a visual stimulus, children and adolescents are more likely than adults to perceive a wider range of onsets as simultaneous (Hillock et al., 2011; Hillock-Dunn \& Wallace, 2012; Lewkowicz \& Flom, 2014). However, for complex stimuli such as audiovisual speech, the temporal binding window may narrow to adult-like levels earlier in childhood (Hillock-Dunn et 
al., 2016). This raises the possibility that if the auditory advantage arises in part from sensory differences such as the asymmetrical temporal binding window, children may exhibit a smaller (more adult-like) auditory advantage with complex audiovisual stimuli like music and images than with simple stimuli like flashes and beeps.

It is currently unknown if children exhibit an auditory advantage for beat and meter processing. Rhythmic structure is perceptually salient early in life: young infants use culturespecific rhythmic information to distinguish between melodies (Hannon \& Johnson, 2005; Hannon \& Trehub, 2005) and between spoken languages (Nazzi et al., 1998; Jusczyk et al., 1993). While infants are initially able to discriminate rhythms from any culture, they begin to respond preferentially and show better discrimination of rhythms from their own culture from 612 months of age (Hannon et al., 2011; Hannon \& Trehub, 2005). Children gradually improve at beat perception and synchronization with music throughout childhood and adolescence (Drake, Jones, et al., 2000; Einarson \& Trainor, 2016; Nave-Blodgett et al., 2021; Puyjarinet et al., 2017). With increasing age, culture-specific biases become more robust (Hannon, Soley, et al., 2012; Hannon, Vanden Bosch der Nederlanden, et al., 2012). This evidence underscores the critical role of listening experiences in the development of beat perception, but it is unclear whether or not such experience leads to an auditory advantage.

To our knowledge, no study has directly examined if there is an auditory advantage for the perception of multiple levels of musical meter with complex, human-performed musical excerpts rather than simple computer-generated sine-wave rhythms or MIDI melodies. Previous examinations of auditory versus visual beat perception have often used auditory beeps and visual flashes to create rhythms and induce a sense of beat (e.g., Grahn et al., 2011; McAuley \& Henry, 2010; Pasinski et al., 2016), which although tightly controlled, are less ecologically valid than 
real music. Beat and meter perception in everyday listening requires listeners to determine the beat location in music containing many overlapping and continuous instrumental lines articulating different non-isochronous rhythms, rather than a simple event-onset-to-beat mapping (van der Weij et al., 2017). We used commercially recorded ballroom dance music for our stimuli as they were specifically composed with the intention of eliciting the perception of and synchronization with multiple metrical levels and contains many acoustic cues to meter. The rhythmic and metrical structures in the ballroom dance music were also culturally familiar exemplars for our participants.

The current study had two goals. First, we asked whether individuals demonstrate an auditory advantage for beat and meter. Second, we asked if this potential auditory advantage for meter perception is present early in life or increases with age and with music or dance experience. We adapted a test of meter perception (Nave-Blodgett et al., 2021) that paired complex, ecologically-valid musical excerpts with auditory, visual, and audiovisual metronomes reflecting two metrical levels. Participants performed a simple task where they rated the fit between the music and the metronomes. We used this task to compare auditory and visual meter perception in adults both across (Experiment 1) and within (Experiment 2) individuals, and in typically-developing children (Experiment 3). We recruited large, diverse samples with wide ranges of formal music and dance training to examine the effects of varying amounts of experience on perception. All data, stimulus materials, experimental programs, and analysis scripts are publicly available in a repository at the Open Science Foundation: https://osf.io/xgsvk/ (Nave-Blodgett et al., 2021).

\section{Experiment 1}


In Experiment 1, adult participants with widely varied backgrounds of formal music and dance training rated the fit between musical excerpts and auditory, visual, or audiovisual metronomes that matched or mismatched multiple levels of metrical structure (i.e., the beat and the measure). We included the audiovisual metronome condition to see if participants would benefit from multimodal over unimodal information in the metronomes.

\section{Method}

\section{Participants}

Ninety individuals (61 female) participated in the study and received credit in psychology classes at the University of Nevada, Las Vegas for their participation. We compared these data to the results of 39 individuals who participated in an auditory metronome condition, which were collected a few months prior, and previously reported in Nave-Blodgett et al. (2021). The resulting combined dataset contained 129 (85 female) participants. Demographic information for participants in each metronome modality and data set are provided in Table 1 . We did not specifically recruit or exclude individuals based on their musical or dance training. All participants in both datasets self-reported normal hearing and normal or corrected-to-normal vision, and no history of uncorrected hearing or visual problems. All participants in both datasets spoke English, either having learned English as their first language $(n=93)$ or during childhood ( $n=36, M_{\text {age learned }}=4.30$ years, $S D=2.16$ years, range: $1-9$ years $)$. Eight additional participants began the task, but their data were lost due to experimenter error $(n=4)$ or program error $(n=4)$. 
Table 1. Participant Demographic Information - Experiments 1 and 2

\begin{tabular}{|c|c|c|c|c|c|c|}
\hline \multirow[b]{2}{*}{ Metronome Modality } & \multicolumn{5}{|c|}{ Experiment 1} & \multirow{2}{*}{$\begin{array}{c}\text { Experiment } 2 \\
\text { Auditory and } \\
\text { Visual }\end{array}$} \\
\hline & $\begin{array}{l}\text { Full Sample } \\
\text { (combined) }\end{array}$ & $\begin{array}{l}\text { Visual - } \\
\text { Upright }\end{array}$ & $\begin{array}{l}\text { Visual - } \\
\text { Tilted }\end{array}$ & Audiovisual & $\begin{array}{l}\text { Auditory } \\
\text { (Nave- } \\
\text { Blodgett et } \\
\text { al., 2021) }\end{array}$ & \\
\hline$n$ ( $n$ Females $)$ & $129(85)$ & $28(17)$ & $32(23)$ & $30(21)$ & $39(24)$ & $32(18)$ \\
\hline Age in Years & $\begin{array}{c}20.31 \\
( \pm 3.53)\end{array}$ & $\begin{array}{c}19.46 \\
( \pm 1.33)\end{array}$ & $\begin{array}{c}20.42 \\
( \pm 3.23)\end{array}$ & $\begin{array}{c}19.66 \\
( \pm 1.70)\end{array}$ & $\begin{array}{c}21.28 \\
( \pm 5.22)\end{array}$ & $26.31( \pm 8.24)$ \\
\hline Age Range & $18-45$ & $18-23$ & $18-34$ & $18-25$ & $18-45$ & $18-50$ \\
\hline$n$ Hispanic $(\%)$ & $32(25 \%)$ & $7(25 \%)$ & $8(25 \%)$ & $9(30 \%)$ & $8(21 \%)$ & $6(19 \%)$ \\
\hline$n$ bilingual (\%) & $49(38 \%)$ & $8(29 \%)$ & $13(41 \%)$ & $17(57 \%)$ & $11(28 \%)$ & $11(34 \%)$ \\
\hline$n$ Non-English L1 (\%) & $36(28 \%)$ & $9(32 \%)$ & $8(25 \%)$ & $10(33 \%)$ & $9(23 \%)$ & $7(22 \%)$ \\
\hline $\begin{array}{l}n \text { Parents' Highest Education } \\
\text { Level } 4 \text {-Year College degree or } \\
\text { higher }(\%)\end{array}$ & $57(44.2 \%)$ & $13(46 \%)$ & $13(41 \%)$ & $12(40 \%)$ & $19(49 \%)$ & $19(59 \%)$ \\
\hline $\begin{array}{l}n \text { Participants with Music } \\
\text { Training }(\%)\end{array}$ & $60(47 \%)$ & $12(42.9 \%)$ & $15(47 \%)$ & $15(50 \%)$ & $19(49 \%)$ & $22(69 \%)$ \\
\hline Years Music Training & $2.13( \pm 3.05)$ & $1.68( \pm 2.72)$ & $2.31( \pm 3.15)$ & $2.17( \pm 3.08)$ & $2.28( \pm 3.25)$ & $10.03( \pm 12.00)$ \\
\hline Range of Music Training & $1.0-15.0$ & $1.0-10.0$ & $1.0-10.0$ & $0.5-13.0$ & $1.0-15.0$ & $1.0-40.0$ \\
\hline $\begin{array}{l}n \text { Participants with Dance } \\
\text { Training }(\%)\end{array}$ & $36(28 \%)$ & $7(25 \%)$ & $10(31 \%)$ & $9(30 \%)$ & $10(26 \%)$ & $8(25 \%)$ \\
\hline Years Dance Training & $1.49( \pm 3.54)$ & $1.18( \pm 3.65)$ & $2.13( \pm 4.07)$ & $1.10( \pm 2.26)$ & $1.49( \pm 3.84)$ & $0.98( \pm 2.91)$ \\
\hline Range of Dance Training & $1.0-17.0$ & $0.1-15.0$ & $1.0-16.0$ & $1.0-10.0$ & $1.0-17.0$ & $0.5-15.0$ \\
\hline Hours Music Listening/Week & $\begin{array}{c}16.06 \\
( \pm 11.44)\end{array}$ & $\begin{array}{c}13.77 \\
( \pm 13.98)\end{array}$ & $\begin{array}{c}15.58 \\
( \pm 12.19)\end{array}$ & $\begin{array}{c}15.35 \\
( \pm 18.02)\end{array}$ & $\begin{array}{c}18.91 \\
( \pm 10.85)\end{array}$ & $15.56( \pm 14.29)$ \\
\hline
\end{tabular}

Note: All values are means and standard deviations unless otherwise specified. Years music training and years dance training include all participants in the sample. Ranges of music and dance training only include participants with relevant training. All values are based on participant self-report. 


\section{Power Analysis}

Our aim was to detect, if present, significant interactions between beat-level or measurelevel sensitivity and metronome modality (e.g., beat x modality, beat x measure x modality; cross-level interactions). Auditory-only metronome-matching tasks containing beat-level metronomes have reported large effect sizes ranging from $\mathrm{d}=1.27$ to 1.70 (Leow et al., 2014; Müllensiefen et al., 2014). Studies comparing auditory to visual beat perception have reported effect sizes for modality differences (both main effects of modality and interactions with beat or rhythm) from lower estimates of $\eta_{p}^{2}$ of .22 to .28 (Pasinski et al., 2016), .26 to .32 (McAuley \& Henry, 2010), or as high as $\eta_{p}^{2}$ of .64 to .68 (Grahn et al., 2011). A study comparing audiovisual to auditory-only beat perception found a $\eta_{p}^{2}$ of .57 for modality $(\mathrm{Su}, 2014)$. These effect sizes roughly translate to standardized effect sizes (Cohen's $d$ ) of 1.0 - 2.0 (Fritz et al., 2012).

We conducted a power analysis using the "Optimal Design Plus Empirical Evidence" program (Raudenbush \& Liu, 2000; Spybrook et al., 2011) to estimate sample size for our multilevel model analytical approach where trials (level 1) were clustered within participants (level 2), and modality was manipulated among participants (level 2). We estimated smaller effect sizes than previously reported to maximize our chances of detecting a real effect, if present. We modeled the required sample size (number of participants) to achieve a power of 0.8 to detect effect sizes between $d=0.20$ and $d=0.35$ for fixed effects of cross-level interactions, and estimated the intra-class correlation (ICC) between $0.10-0.15$. At $d=0.20$ and ICC of 0.15 (the most conservative estimate), a sample size of 128 participants with 96 trials/participant was required for 0.8 power. For larger effect sizes $(d=0.25-0.35)$ and smaller ICCs, sample sizes ranging from 30-80 participants were sufficient to reach 0.8 power. Our sample size of 129 individuals with 96 trials each was determined to be adequate for assessing small-to-moderate 
fixed effects of cross-level interactions. Other advice for sample size estimates in multilevel model designs suggest adhering to a 30/30 rule: a minimum of 30 level 2 clusters (participants) and 30 level 1 measurements (trials; Hox, 1995, 2010) to achieve reasonable power in multilevel modeling. Our sample size and trial number together exceeded this threshold.

\section{Stimuli}

The stimuli in Experiment 1 consisted of musical excerpts (from Nave-Blodgett et al., 2021) paired with auditory, visual, and audiovisual metronomes that matched or mis-matched the beat and measure locations in the music. Stimuli can be found at: https://osf.io/xgsvk/ (NaveBlodgett et al., 2021).

The six musical excerpts were drawn from commercially available ballroom dance music (compact disc, "Ballroom Dance Music," the Swiss Ballroom Orchestra, Blaricum CD company, 1999). Half of the musical excerpts were in duple meter (4/4) and half in triple meter (3/4). We matched the musical stimuli into pairs of duple and triple meter at slow ( 89 beats per minute [BPM]), medium (104 BPM), and fast (124 BPM) tempi. We further equated the tempo of the stimuli in each pair using the "Change Tempo" function in Audacity® (version 2.1.2; Audacity Team, 2021), which did not alter the pitch of the music. From each musical piece, we extracted four five-measure excerpts for use in the experiment, for a total of 24 musical excerpts (six musical pieces with four excerpts each). These musical excerpts ranged from 7-14 seconds in duration (depending on tempo).

We determined the beat and measure locations in each musical excerpt using the Bar and Beat Tracker VAMP plugin (Davies \& Plumbley, 2007; Stark et al., 2009) executed in Audacity®, and checked by the first author (a trained musician). From these time points, we created four possible metronome alignment conditions. The fully-matching metronome had 
measure-level and beat-level ticks that aligned with the measure and beat of the music (Figure

2A). The beat-matching measure-mismatching metronome had beat-level ticks that aligned with the beat of the music, but measure-level ticks that misaligned with the measure (and matched the wrong meter, for example occurring every three beats instead of every four beats) (Figure 2B). The beat-mismatching measure-matching metronome had measure-level ticks that aligned with the measure of the music, but beat-level ticks that were misaligned with the musical beat (and were in the wrong meter; Figure 2C). The fully-mismatching metronome was in the wrong meter (e.g. triple for duple and vice versa) and the tempo of the metronome was $6 \%$ faster than the music (Figure 2D). All metronome conditions were created by hand by the first author.

To account for possible stimulus-driven effects in the absolute time-differences between beat-level and measure-level ticks in the metronome versus the music for each individual excerpt, we calculated the absolute asynchrony (leading/following) between the metronome ticks and the beat and measure of the music for each musical excerpt/metronome pairing. We included this asynchrony value for each individual stimulus as a random factor in our analyses to account for the relative impact of differences between metronome ticks and musical excerpt tempi.

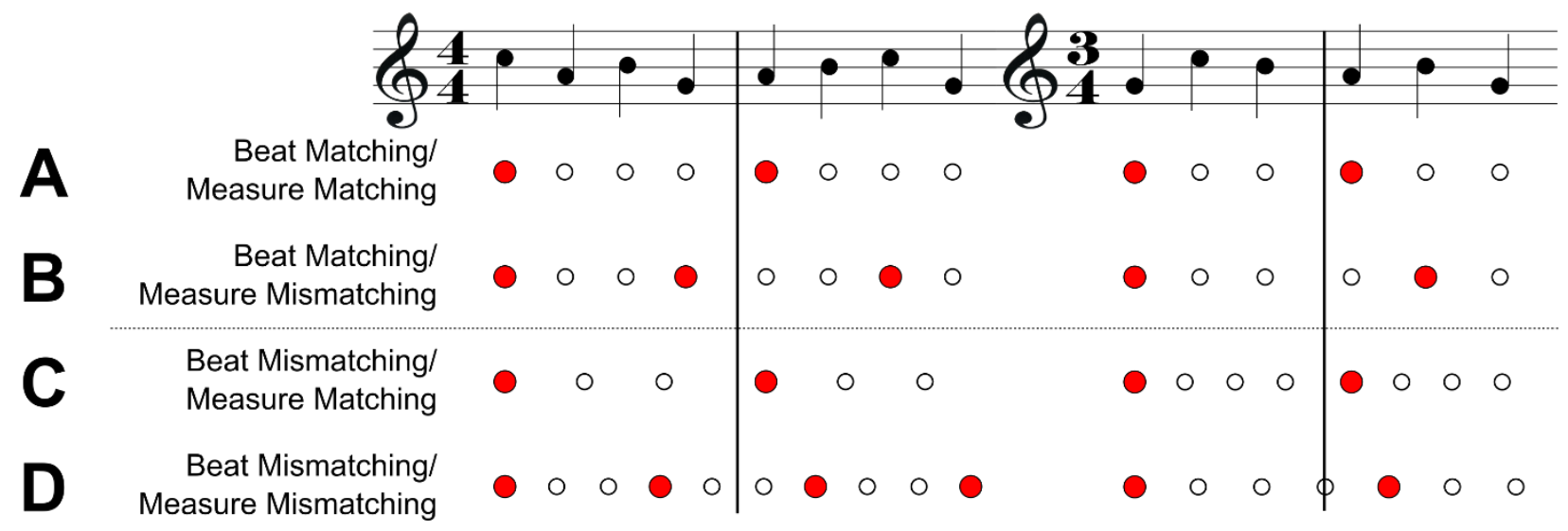

Figure 2. Metronome conditions relative to the meter of the musical excerpts. Measure-level ticks are indicated by filled (red) circles, and beat-level ticks by empty (white) circles. Vertical alignment between a musical note and a circle indicates onset synchrony between musical and 
metronome events; vertical misalignment indicates asynchrony between musical and metronome events. (A) Fully-matching (B) Beat-matching/Measure-mismatching (C) Beatmismatching/measure-matching (D) Fully mismatching.

Metronomes were auditory, visual, or audiovisual. Auditory metronomes consisted of 10millisecond sine-wave ticks with no on- or off-ramp. This intentionally created a "click" sound that did not blend with the musical excerpt, was unlikely to be mistaken for a musical instrument, and remained perceptually separate from the texture of the musical excerpt. Measure-level ticks had a higher pitch $(\mathrm{G \# 6;1661.2} \mathrm{Hz)} \mathrm{than} \mathrm{beat-level} \mathrm{ticks} \mathrm{(G \# 5,} \mathrm{830.6} \mathrm{Hz).} \mathrm{Throughout} \mathrm{the} \mathrm{trial,}$ the screen was uniformly black except for white text in the center of the screen that said "Listen..."

The visual metronomes consisted of a white circle with a black outline, resembling a clock-face without numbers, presented on a white background (Figure 3). Each metronome was constructed from either three or four static images. Ticks in the visual metronome were marked with an arrow that was anchored at the center of the circle and extended to the edge of the circle, like a clock hand. The arrow was wider and red at measure-level ticks, and narrower and black for beat-level ticks. The position of the arrow divided the circle into quarters for metronomes in duple meter or thirds for metronomes in triple meter. There were two alignments of the visual metronome: upright and tilted. We created the tilted version of the duple metronome to make sure that any possible differences observed in sensitivity to duple versus triple meter in the metronome were not due to the lines being horizontal or vertical (duple) versus oblique (triple): in the tilted version, both triple and duple metronomes included oblique lines. For the upright version, the duple beats were marked at 12:00 (downbeat), 3:00, 6:00, and 9:00 (Figure 3A). In the tilted version, the duple beats were spaced at 1:30 (downbeat), 4:30, 7:30, and 10:30 (Figure 
3B). The triple meter metronome beats were marked at 12:00, 4:00, and 8:00 in both upright and tilted conditions. Each frame was presented on-screen without change until the next beat of the metronome occurred, giving the impression of discrete motion and the arrow jumping from point to point (as in Grahn, 2012).

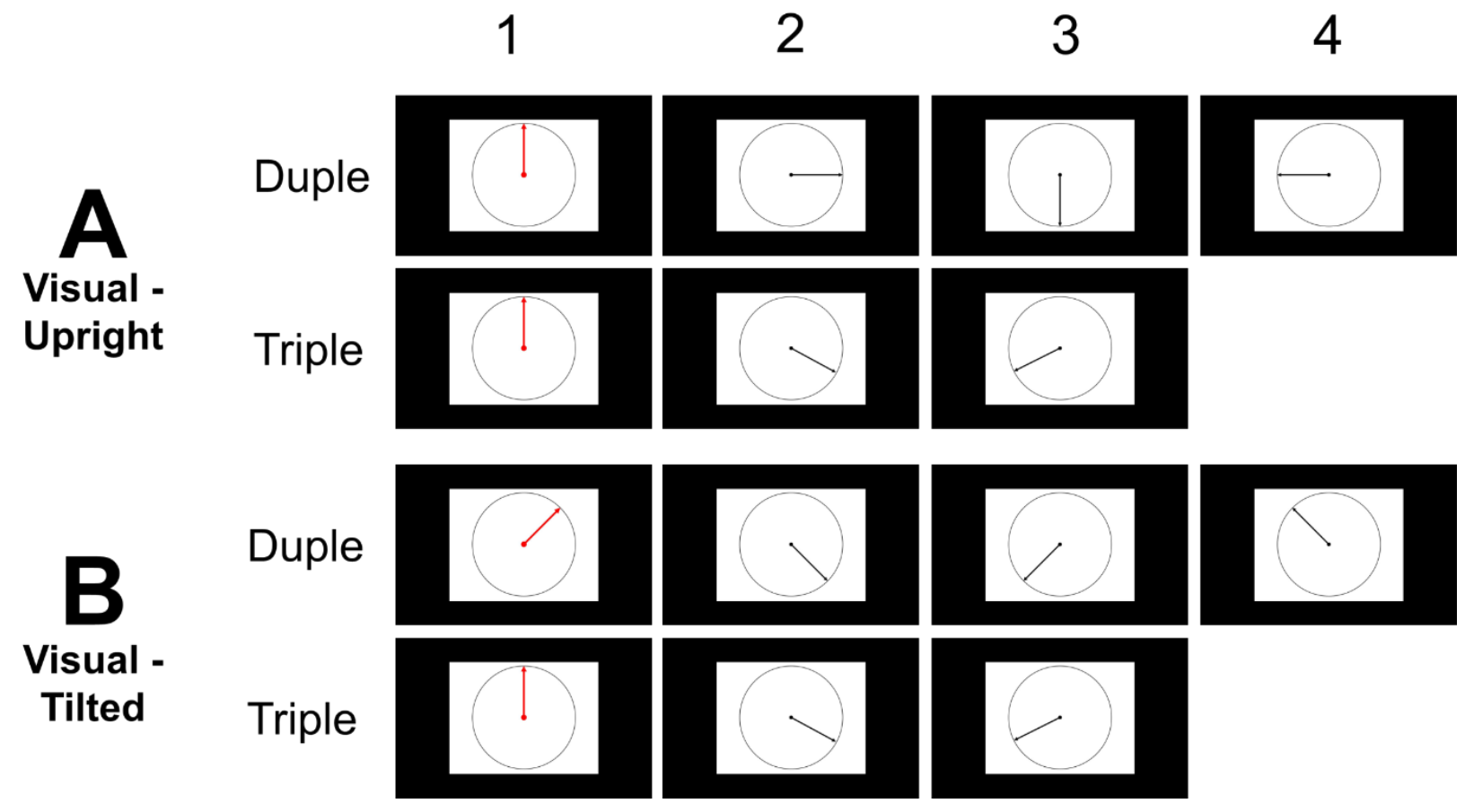

Figure 3. Individual frames used in the visual and audiovisual metronome conditions. All visual images were presented in the center of a computer monitor with a black background. (A) The "upright" visual metronome condition. (B) The "tilted" visual metronome condition, with the duple meter metronome at a $45^{\circ}$ offset from "upright." The triple meter metronome is identical in both visual versions. The audiovisual metronome condition used the same images as the "upright" (panel A) visual condition, such that the auditory metronome "ticks" were presented simultaneously with onsets of the visual metronome frames.

On each trial in the visual metronome condition, participants heard the musical excerpt while the metronome was presented in the center of the computer screen against a black background. Each metronome frame was 960 x 720 pixels in size and presented on an LCD monitors with a screen resolution of 1680 x 1050 pixels. Participants sat approximately $70 \mathrm{~cm}$ 
away from the computer screen, and the metronome and surrounding frame subtended a visual angle of approximately $15.5^{\circ}$.

The audiovisual metronome condition contained both visual (upright; Figure 3A) and auditory ticks, presented simultaneously. The possible inter-tick intervals in the metronomes (auditory and visual) ranged from 363 - $899 \mathrm{~ms}$. Because of occasional misalignment with the monitor frame rate $(60 \mathrm{~Hz})$, a visual metronome tick could be jittered by a maximum of $\pm 16.6 \mathrm{~ms}$ compared to the audio metronome tick, or compared to the location of the beat in the musical excerpt. This would have been perceived as simultaneous by participants as this stimulus-onset asynchrony is below the 30-ms cross-modal temporal order and simultaneity thresholds for audition and vision (van Wassenhove, 2009), and within the temporal binding window of $100 \mathrm{~ms}$ (for both audio leading visual and vice versa; Stevensen, Zemtsov, et al., 2012).

All participants were presented with 96 unique trials (6 musical pieces, 4 excerpts per musical piece, and 4 metronome/musical alignment conditions), regardless of the metronome modality condition. Meter (3/4 and 4/4) and tempo (89, 104, and 124 BPM) of the musical stimuli were equally balanced across trials. All auditory stimuli were presented binaurally: when the metronome was visual, the musical excerpts were presented diotically. When there was an auditory metronome (auditory-only or audiovisual metronome conditions), the auditory metronome was presented in a separate channel from the music (e.g., musical excerpt in right ear, metronome in left ear) to facilitate stream segregation rather than integration of the metronome and music (Hartmann \& Johnson, 1991). Half of the auditory metronomes were presented in the right ear and half in the left ear.

\section{Apparatus and Procedure}


Participants' task in this experiment was to rate how well the metronome fit the music. The participants performed the task on a desktop computer (Dell, Round Rock, TX) running Windows 7 (Microsoft Corporation, Seattle, WA), wearing over-the-ear noise-attenuating headphones (approximately 20dB SPL noise attenuation; Sennheiser 280 Pro, Sennheiser Corporation, Old Lyme, CT). All stimuli were presented to participants at approximately $70 \mathrm{~dB}$ SPL, well above normal hearing thresholds. A custom experimental program coded in Presentation software (Neurobehavioral Systems, Palo Alto, CA) controlled the presentation of stimuli and recording of responses.

Participants were assigned to a metronome modality condition (visual-upright, visualtilted, audiovisual) upon arrival to the lab, with different metronome modalities tested on different days. All participants provided informed consent to participate in the experiment prior to beginning the task. Instructions for the rating task were presented orally (by the experimenter) and on-screen, in English. For the auditory condition, the instructions stated, "On each trial, you will hear a clip of music in one ear and a click track in the other ear." The visual versions amended this to "...hear a clip of music and see a click track on the monitor." The audiovisual version stated "...hear a clip of music in one ear and hear and see a click track on the monitor." All versions continued with "We would like you to tell us how well the click track matches the music you hear.” On each trial, participants listened to a musical excerpt paired with a metronome for five full measures of music. Participants were then prompted to enter their rating of fit for how well the metronome matched the music on a Likert-type scale from " 1 " ("Not Well at All") to "4" ("Very Well"). The prompt to respond only appeared after the entire excerpt had played, and the program only accepted responses entered after the prompt. Each trial ended after participants responded or after five seconds elapsed without a response. 
All participants completed a practice session. Participants first encountered three demonstration trials that contained musical excerpts not used in the experiment paired with a fully-matching metronome, a beat matching/measure mismatching metronome, and a fullymismatching metronome. Each pair was followed by text stating how other listeners might rate this music/metronome pair, e.g., "Most people would think is an example of a not very well matching click track," for a fully-mismatching metronome, etc. Participants then completed three practice trials during which they entered fit ratings and received the same information as in the demonstration trials. After the practice session, participants could ask the experimenter for clarification on the task or begin the experimental trials. The experiment contained four blocks of 24 trials each, presented in a random order for each participant. Between every block of trials, participants had the opportunity to take a short break for a maximum of five minutes while remaining seated at the computer.

After completing the computer-based portion of the experiment, participants filled out a brief demographic questionnaire about their musical, dance, language, and cultural background. The entire experiment lasted approximately 30 minutes, including informed consent, practice, test trials, and demographic questionnaire. All procedures were approved by the Institutional Review Board (IRB) of the University of Nevada, Las Vegas, and all participants were treated in an ethical manner consistent with the APA Code of Ethics.

\section{Data Analysis}

We investigated how differences in metronome modality and beat- and measure-level matching between the metronome and music affected participants' ratings of fit, while controlling for musical tempo, metronome asynchrony, and meter differences for each trial within participants and the possible influences of music training, dance training, and musical 
listening between participants. To do this, we used multilevel linear modeling (MLM). MLM analyses of repeated-measures (within-participants) designs allow the simultaneous investigation of within-participant experimental manipulations (e.g., beat and measure matching) while accounting for between-participant individual differences (e.g., formal music or dance training) and the cross-level interactions of the two while also allowing researchers to account for stimulus-level characteristics and differences between trials (Baayen et al., 2008; Hox, 2010; Raudenbush \& Bryk, 2002).

We had three main hypotheses. First, we predicted that beat- and measure-level alignment between metronome and music would significantly impact participants' ratings, such that participants would give the highest ratings to metronomes that matched the music at two metrical levels (a beat x measure interaction). Second, we hypothesized that metronome modality (auditory, audiovisual, and visual) would interact with beat- and measure-level sensitivity. We expected an auditory advantage for beat-level and possibly for measure-level perception (modality $\mathrm{x}$ beat, modality $\mathrm{x}$ measure, and modality $\mathrm{x}$ beat $\mathrm{x}$ measure interactions). Third, we hypothesized that music and dance training would moderate beat- and measure-level sensitivity, and this might vary by metronome modality, with dancers more sensitive to visual metronomes and musicians more sensitive to auditory metronomes (beat $\mathrm{x}$ training, measure $\mathrm{x}$ training, modality $\mathrm{x}$ training, and beat $\mathrm{x}$ modality $\mathrm{x}$ training or measure $\mathrm{x}$ modality $\mathrm{x}$ training interactions).

We tested these hypotheses using two models. In Model 1, we tested the first and second hypotheses by including the within-subjects variables of beat (matching or mismatching), measure (matching or mismatching), tempo (slow, medium, fast), and musical meter (duple or triple), along with the interaction terms and the between-subjects variables of metronome 
modality (visual, audiovisual, or auditory), music training, dance training, and music listening as fixed effects. We included random effects for beat-level and measure-level asynchrony between each musical excerpt and metronome (in seconds) to control for the possible influence of differences in beat- and measure-level asynchrony based on metronome alignment to music. In Model 2, we tested the third hypothesis by adding cross-level interactions among beat, measure, modality, and training. Both models were compared to an unconditional (baseline) model, equivalent to a one-way ANOVA with participant (subject) as the random effect. Participants' ratings of how well the metronome fit the music in each trial was the dependent variable in all models.

All within-participants (level 1) predictors were dichotomous variables. Beat and measure were coded as 0 for mismatching and 1 for matching. Tempo was dummy coded into two dichotomous variables, slow (89 BPM) and fast (124 BPM), with the moderate (104 BPM) tempo used as the reference condition (both values at zero). The meter (time signature) of the musical excerpt was coded with 0 for duple meter (4/4) and 1 for triple meter (3/4). The between-subjects factor of metronome modality was separated into two dummy-coded dichotomous variables: audiovisual and visual, with the auditory modality used as the reference condition (both values at zero) ${ }^{1}$. All other between-participants (level 2) predictors were grandmean centered: the grand mean of all participants for each variable (music /dance training, music

\footnotetext{
${ }^{1}$ During the model-building process, we did not find any significant differences between the ratings of fit for the Visual-Upright and Visual-Tilted metronome conditions. Thus for all analyses we combined the Visual-Upright and Visual-Tilted metronome conditions into a single "Visual" modality condition.
} 
listening) was calculated, and then subtracted from each participant's score on the relevant variable. We did not standardize between-participant predictors. The values for the random effects of beat- and measure-level asynchrony consisted of the absolute difference between the temporal location of a beat (or measure-level beat) in the musical excerpt and the beat or measure tick in the metronome. There were four possible metronome alignments for each of six musical excerpts, resulting in 24 unique values for each variable (beat asynchrony range: 0.15 212.16 ms; measure asynchrony range: $0.15-666.58 \mathrm{~ms}$; values were in seconds in the analyses). We treated all missing data as data missing-completely-at-random (MCAR), as there was no increased likelihood of missing a response as a function of missing a previous response (Rubin, 1987). Each participant experienced 96 trials in the experiment. However, as the experiment moved on automatically to the next trial after 5 seconds with no response, there were a small number of missing trials across the entire dataset $(11$ trials; $<0.01 \%)$. Eight individuals had at least one missed trial, and three individuals missed two trials ( $2 \%$ of an individual's total). We did not impute missing values for ratings of fit (dependent variable) and removed those trials from the analysis. All values were present for within-participants factors. Most betweenparticipants (Level 2) data were present, but nineteen participants did not provide information about hours of music listening: we imputed the mean for these missing between-participant factors.

Statistical analyses were performed in R version 4.0.3 (R Core Team, 2020). We used the package lme4 (Bates et al., 2015) for linear mixed-effects models, and the package lmerTest (Kuznetsova et al., 2017) to estimate degrees of freedom and calculate two-tailed $p$-values for predictors and interaction terms using Satterthwaite approximations (Satterthwaite, 1946). All models used restricted maximum likelihood estimation (REML), unstructured covariance 
matrices, and allowed for covariance between random slopes and intercepts. The dependent variable (participants' ratings of fit) was treated as Gaussian in distribution. Simple-slopes and slopes-comparisons tests of interaction terms were performed using the methods specified in Preacher et al. (2006) and Dawson and Richter (2006). Data visualizations were created using ggplot2 (Wickham, 2016). All models presented successfully converged. Datasheets and R code are available on the OSF repository for this paper.

\section{Results and Discussion}

Ratings of fit by metronome are shown in Figure 4. Ratings ranged from 1 (poor fit) to 4 (excellent fit). MLM results, including coefficients, estimated variances, and intraclass correlations (ICC), are reported in Table 2. In the baseline (intercept-only) model, most of the variance in ratings was attributable to within-subjects variance $(1.26,95 \% \mathrm{CI}: 1.23-1.29)$, and a small portion was attributable to between-subjects variance $(0.06,95 \% \mathrm{CI}: 0.05-0.08)$. Approximately $95 \%$ of the unexplained variance was within-participants (experimental effects) and $5 \%$ between-participants (individual differences). 


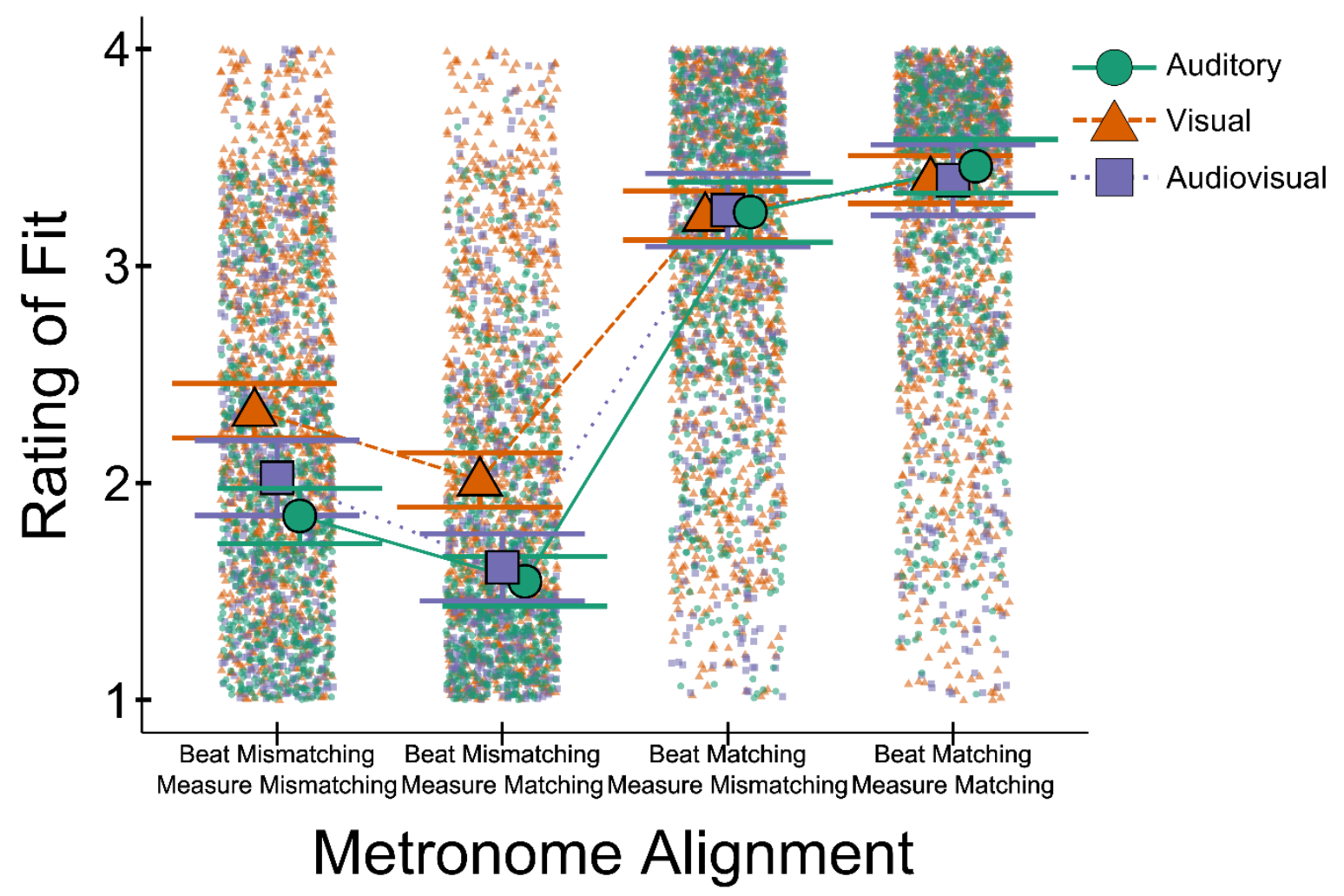

Figure 4. Ratings of fit between metronome and music by beat- and measure-level alignment. Lower ratings indicate poorer perceived fit, higher ratings indicate better perceived fit.

Responses could range from 1 through 4 in integers: vertical and horizontal jitter has been added to the plot to facilitate visualization. All responses are shown (approximately 12,000), with the shape (and color) of each point indicating the metronome modality, and relative opacity and density indicating more (or fewer) responses ( $n=129$ participants, 96 responses per participant). Group means represent the average rating and the $95 \%$ confidence interval of the mean for each of the three metronome modalities: auditory $(n=39)$, audiovisual $(n=28)$, visual $(n=60)$. Please refer to the online version of this article to see the color version of this figure. 
Table 2. Results of Multilevel Models in Experiment 1 Investigating Differences in Ratings of Fit of Metronome to Music as a function of Within- and Between-Participant Factors

\begin{tabular}{|c|c|c|c|}
\hline Parameter & Baseline & Model 1 & Model 2 \\
\hline \multicolumn{4}{|c|}{ Fixed Effects } \\
\hline Average Rating of Fit (Intercept) & $2.638 * *$ & $1.863^{* *}$ & $1.863 * *$ \\
\hline \multicolumn{4}{|l|}{ Within-Subjects Factors (Level 1) } \\
\hline Beat & & $1.361 * *$ & $1.363 * *$ \\
\hline Measure & & $-0.281 * *$ & $-0.283 * *$ \\
\hline Slower Tempo & & $0.053 * *$ & $0.051 * *$ \\
\hline Faster Tempo & & $0.163^{* *}$ & $0.164 * *$ \\
\hline Musical Meter & & $-0.158 * *$ & $-0.159 * *$ \\
\hline Beat $\mathrm{x}$ Measure & & $0.501 * *$ & $0.505^{* *}$ \\
\hline Beat x Visual Modality & & $-0.600 * *$ & $-0.578 * *$ \\
\hline Measure x Visual Modality & & -0.001 & 0.000 \\
\hline Beat x Measure x Visual Modality & & -0.056 & -0.053 \\
\hline Beat x Audiovisual Modality & & -0.199 & $-0.246^{*}$ \\
\hline Measure x Audiovisual Modality & & -0.112 & -0.107 \\
\hline Beat x Measure x Audiovisual Modality & & 0.030 & 0.023 \\
\hline Beat x Music Training & & & 0.027 \\
\hline Measure x Music Training & & & 0.011 \\
\hline Beat $\mathrm{x}$ Measure $\mathrm{x}$ Music Training & & & -0.016 \\
\hline Beat $x$ Dance Training & & & -0.010 \\
\hline Measure x Dance Training & & & $0.033^{*}$ \\
\hline Beat $\mathrm{x}$ Measure $\mathrm{x}$ Dance Training & & & $-0.018^{*}$ \\
\hline Visual Modality x Music Training & & & $-0.062 *$ \\
\hline Audiovisual Modality x Music Training & & & -0.046 \\
\hline Visual Modality x Dance Training & & & -0.025 \\
\hline Audiovisual Modality x Dance Training & & & 0.064 \\
\hline Beat x Visual Modality x Music Training & & & 0.040 \\
\hline Measure x Visual Modality x Music Training & & & $0.038^{*}$ \\
\hline Beat x Audiovisual Modality x Music Training & & & $0.083^{*}$ \\
\hline Measure x Audiovisual Modality x Music Training & & & 0.015 \\
\hline Beat x Visual Modality x Dance Training & & & 0.026 \\
\hline Measure x Visual Modality x Dance Training & & & 0.004 \\
\hline Beat x Audiovisual Modality x Dance Training & & & $-0.125^{* *}$ \\
\hline Measure x Audiovisual Modality x Dance Training & & & -0.024 \\
\hline \multicolumn{4}{|l|}{ Between-Subjects Factors (Level 2) } \\
\hline Visual Modality & & $0.526 * *$ & $0.513 * *$ \\
\hline Audiovisual Modality & & $0.191 *$ & $0.209 *$ \\
\hline Music Training & & 0.005 & -0.001 \\
\hline Dance Training & & -0.001 & -0.006 \\
\hline Music Listening & & -0.001 & -0.001 \\
\hline \multicolumn{4}{|c|}{ Random Effects } \\
\hline Within-Subjects (Residual) & $1.263^{* *}$ & $0.643 * *$ & $0.642 * *$ \\
\hline Between-Subjects (Intercept) & $0.063 * *$ & $0.181 * *$ & $0.154 * *$ \\
\hline Intraclass Correlation (ICC) & 0.047 & 0.219 & 0.194 \\
\hline
\end{tabular}


Note. All coefficients are unstandardized. $* p<.05, * * p<.01$

\section{Hypothesis 1: Participants Perceive Beat, Measure, and Meter in All Modalities}

Model 1 tested the first and second hypotheses by examining the effects of the withinsubjects (Level 1) experimental factors of beat, measure, the beat x measure interaction, and the between-subjects (Level 2) manipulation of metronome modality (audiovisual and visual), and the cross-level interactions of beat x modality and measure x modality on participants' ratings. We controlled for the between-subjects factors of music training, dance training, and music listening as fixed effects, and incorporated random slopes for beat-level and measure-level asynchrony between metronome and music to control for stimulus-level variability.

Beat had a stronger effect than measure on participants' ratings, but participants responded to matching and mismatching at both metrical levels. Participants gave higher ratings to beat-matching metronomes compared to beat-mismatching metronomes when controlling for all other factors $(\gamma=1.36,95 \% \mathrm{CI}: 1.29-1.43)$, yet they rated measure-matching metronomes as slightly worse-fitting than measure-mismatching metronomes $(\gamma=-0.28,95 \%$ CI: $-0.33--0.23)$.

This counter-intuitive effect of measure can be interpreted in light of the significant beat $\mathrm{x}$ measure interaction, which demonstrates that beat-level alignment between the metronome and music moderates the response to measure-level matching. A simple-slopes test revealed that when the beat of the metronome matched the music, participants gave higher ratings to measurematching metronomes $(M=3.44,95 \% \mathrm{CI}: 3.34-3.55)$ than to measure-mismatching metronomes $(M=3.22,95 \%$ CI: $3.09-3.36)$, while controlling for other factors $(\omega=0.22,95 \%$ CI: $0.12-0.32)$. In contrast, when the beat of the metronome mismatched the music, participants 
gave lower ratings to measure-matching $(M=1.58,95 \% \mathrm{CI}: 1.47-1.69)$ than measuremismatching $(M=1.86,95 \%$ CI: $1.74-1.98)$ metronomes $(\omega=-0.28,95 \%$ CI: $-0.39--0.17)$. Participants likely found constant beat-level asynchrony more salient than occasional measurelevel synchrony, especially when the two were in conflict.

Supporting this interpretation, the random effects of beat-level and measure-level asynchrony between metronome ticks and musical locations were both significant $(p<.001)$. Both had negative correlations with individual differences in ratings $\left(r_{\text {beat-level asynchrony,intercept }}=-\right.$ $\left..80 ; r_{\text {measure-level asynchrony,intercept }}=-.34\right)$, but the relationship was much stronger for beat-level asynchrony than measure-level asynchrony. This may explain why the beat mismatching/measure matching condition received the lowest ratings of all conditions, since in this condition beat-level asynchrony was highest. Overall, the fact that participants gave the highest ratings to metronomes that matched the music at both the beat and measure levels suggests they could perceive multiple levels of meter simultaneously in all three modality conditions (audio, visual, and audiovisual).

\section{Hypothesis 2: Beat- and Measure-level Sensitivity Differs Across Modalities}

We found evidence of modality differences in beat-level, but not measure-level, perception. There were significant and nearly-significant interactions for beat $\mathrm{x}$ visual modality $(\gamma=-0.60 ; 95 \%$ CI: $-0.69--0.51, p<.001)$ and beat $x$ audiovisual modality $(\gamma=-0.19 ; 95 \%$ CI: $-0.31--0.09, p=.087)$. Beat-level matching affected ratings most in the auditory condition $(\omega=$ 1.36, 95\% CI: $1.21-1.51)$ compared to the audiovisual $(\omega=1.16,95 \% \mathrm{CI}: 0.99-1.34)$ and visual $(\omega=0.76,95 \% \mathrm{CI}: 0.64-0.89)$ conditions, providing evidence of an auditory advantage for beat perception. However, modality differences were only observed for beat-mismatching metronomes, with lower ratings obtained for auditory $(M=1.86)$ than for audiovisual $(M=2.05)$ 
or visual $(M=2.39)$ metronomes. By contrast, ratings for beat-matching metronomes did not differ by modality. We did not observe a significant difference in ratings for measure-level matching as a function of metronome modality, providing no evidence of an auditory advantage for high-level meter perception.

None of the other interactions with metronome modality were statistically significant. After controlling for all other within- and between-participant variables, none of the individual difference variables (music training, dance training, or music listening) had a statistically significant main effect in Model 1.

In Model 1, the addition of the within-subjects predictors accounted for approximately $49 \%$ of the within-subjects variance relative to the random-effects ANOVA baseline model. The proportion of between-subjects variability increased to $22 \%$ and the within-subjects variability decreased to $78 \%$ in Model 1.

\section{Hypothesis 3: Training Relates to Enhanced Meter Perception and Modality Differences}

We assessed the hypothesis that formal training relates to enhanced sensitivity to beatand measure-level information, and this could differ by modality, by adding the cross-level interactions among beat, measure, metronome modality, music training, and dance training to Model 2. All previously significant fixed effects from Model 1 remained statistically significant.

Of the interaction terms added to the model, the beat $\mathrm{x}$ measure $\mathrm{x}$ dance training, measure $\mathrm{x}$ visual modality $\mathrm{x}$ music training, and beat $\mathrm{x}$ audiovisual modality $\mathrm{x}$ both music and dance training interactions reached statistical significance. The beat $\mathrm{x}$ measure $\mathrm{x}$ dance training interaction was small but significant $(\gamma=-0.02,95 \% \mathrm{CI}:-0.03--0.01)$. We explored the interaction with a slopes-comparison test, where we compared the difference in ratings when participants had a larger amount of dance training (+1 SD above the sample mean; 5.03 years 
total) versus no dance training at all, while holding all other terms constant. When the beat of the metronome matched the music, dancers' ratings did not differ for metronomes that did or did not match at the measure level $\left(\omega_{+1 \text { SDdance }}=0.12, p=0.07\right)$, whereas non-dancers gave higher fit ratings to measure-matching than measure-mismatching metronomes $\left(\omega_{\text {nodance }}=0.26, p<.001\right.$; $\left.\omega_{\text {difference }}=-0.14, p=.026\right)$. When the beat of the metronome mismatched, both dancers and nondancers gave higher ratings to measure-mismatching metronomes $\left(\omega_{\text {nodance }}=-0.27, \omega_{+1 \text { SDdance }}=-\right.$ $\left.0.32, \omega_{\text {difference }}=0.05, p=.441\right)$. This suggests that individuals with more dance training were less sensitive to measure-level matching.

The beat $\mathrm{x}$ audiovisual modality $\mathrm{x}$ dance training interaction and the beat $\mathrm{x}$ audiovisual modality x music training interaction were both significant. The first of these interactions arose because there was an auditory (over audiovisual) advantage for beat-level perception for dancers $\left(\omega_{+1 \text { SDdance, auditory }}=1.33, \omega_{+1 \text { SDdance, audiovisual }}=0.64, \omega_{\text {difference }}=-0.68, p<.001\right)$, but not for nondancers $\left(\omega_{\text {no dance, auditory }}=1.32, \omega_{\text {no dance, audiovisual }}=1.38, \omega_{\text {difference }}=-0.06, p=.487\right)$. In contrast, musicians did not show this advantage $\left(\omega_{\text {music,auditory }}=1.44, \omega_{\text {music,audiovisual }}=1.45, \omega_{\text {difference }}=\right.$ $0.01, p>.5)$, while non-musicians $\operatorname{did}\left(\omega_{\text {nomusic,auditory }}=1.31, \omega_{\text {nomusic,audiovisual }}=0.89, \omega_{\text {difference }}=-\right.$ $0.42, p<.001)$

The measure $\mathrm{x}$ visual modality $\mathrm{x}$ music training interaction was also significant. In the auditory metronome condition, musicians $(+1 S D ; 5.18$ years total) and non-musicians both gave lower ratings to measure-matching than measure-mismatching metronomes $\left(\omega_{\text {nomusic }}=-0.31\right.$, $\left.\omega_{+1 \mathrm{SDmusic}}=-0.25, \omega_{\text {difference }}=0.06, p=.450\right)$. In the visual metronome condition, non-musicians gave much lower ratings to measure-matching metronomes than did musicians $\left(\omega_{\text {nomusic }}=-0.40\right.$, $\left.\omega_{+1 \text { SDmusic }}=-0.13, \omega_{\text {difference }}=0.26, p<.001\right)$. Given our interpretation that lower ratings of measure-matching metronomes are driven by greater beat-level asynchronies in the beat- 
mismatching/measure-matching condition, this result may have arisen because musicians were better able to attend to measure-level information and disregard beat-level asynchrony, at least in the visual condition.

No other interactions were significant. The effects of beat-level and measure-level asynchrony remained the same as in Model 1. The addition of the cross-level and level 2 interaction terms in Model 2 accounted for approximately $15 \%$ of the unexplained variance between-subjects in Model 1. Approximately 19\% of the remaining unaccounted-for variance in ratings was between-participants and 81\% was within-participants in Model 2.

\section{Summary}

In all metronome modalities, adults gave the highest ratings of fit to metronomes that matched the music at two metrical levels simultaneously (beat and measure). Beat-level matching robustly influenced ratings in isolation, while the effect of measure-level matching on ratings was moderated by beat-level matching. When the beat of the metronome matched the music, ratings were higher for measure-matching than measure mismatching, but counterintuitively, when the beat of the metronome mismatched the music, lower ratings were given for measure-level matching. We attribute this latter effect to beat-level asynchrony rather than to measure-level alignment or misalignment, since at least in beat-mismatching conditions, beat-level asynchrony was greater for measure-matching than measure-mismatching metronomes. Our interpretation is further supported by the finding that beat-level asynchrony more strongly (and negatively) predicted ratings than did measure-level asynchrony. Sensitivity to beat was greatest in the auditory metronome condition, lower in the audiovisual condition, and lowest in the visual condition. Sensitivity to measure did not differ by modality. 
The finding of lower sensitivity to beat in the bimodal audiovisual versus unimodal auditory metronome condition was somewhat unexpected. Bayesian optimal-integration models suggest individuals weigh and integrate sensory information based on its reliability or accuracy (Alais \& Burr, 2004), and modality-appropriateness models suggest individuals prioritize information from the sense most appropriate to the task at hand and suppress information from less-appropriate senses (Welch et al., 1986). Either model would predict that individuals would weigh the auditory information more heavily than the visual in the audiovisual condition, resulting in comparable performance in the audiovisual and auditory metronome conditions. Instead, performance for the audiovisual condition was poorer than the auditory but better than the visual condition. This suggests that rather than relying on the optimal or most reliable information (auditory), individuals may have weighed the auditory and visual temporal information relatively equally, leading to poorer temporal precision. While it is possible that this effect was driven by the occasional lack of synchronization between the auditory and visual ticks of the audiovisual metronome (maximum $\pm 16.6 \mathrm{~ms}$ ), we do not think this entirely explains our findings as the desynchronization was below the temporal order threshold for audiovisual judgments (van Wassenhove, 2009) and within the multisensory temporal binding window for individuals with and without extensive training (Bidelman, 2016; Stevenson, Wilson, et al., 2013; Stevenson, Zemetsov et al., 2012). Future use of the audiovisual metronomes, paired with music or visual rhythms, could further probe both the tolerance for asynchrony in the audiovisual metronome and the relative weighting of auditory and visual information in making judgments of fit for metrical alignment.

The effects of training were small and differed by type of training. Larger amounts of dance training predicted lower sensitivity to measure in all modalities, and lower sensitivity to 
beat in the audiovisual condition compared to the auditory condition. Greater amounts of musical training predicted similar sensitivity to the beat (and possibly measure) in both auditory and audiovisual metronomes. However, several factors may have contributed to our mixed findings: participants were not specifically recruited for musical or dance training, and thus overall levels of both music $(M=2.13$ years, $S D=3.05$ years $)$ and dance training $(M=1.49$ years, $S D=3.54$ years) were low in this sample. Additionally, as this was a between-subjects design, uneven randomization to metronome modality condition may have weakened our ability to detect any consistent relationships between formal training and modality, especially if the true effects are small or require large amounts of training.

Our finding of an overall auditory advantage for beat perception is in line with previous evidence of auditory superiority for timing tasks. However, we did not find evidence for auditory superiority at the measure level. This could be because there is no auditory advantage for measure-level perception in general, or it could be because pseudo-random assignment to condition cannot eliminate group-level differences that could be obscuring small, but real, modality effects. Across individuals, people vary widely in their sensitivity to beat (Grahn \& Schuit, 2012), but within an individual, beat perception ability is moderately correlated across auditory and visual modalities (Grahn, 2012; Grahn et al., 2011; McAuley \& Henry, 2010; Pasinski et al., 2016). It would be advantageous to compare performance across modalities within the same individuals, which is more statistically powerful, to more conclusively test for an auditory advantage for beat or measure perception.

\section{Experiment 2}

In Experiment 2, we used the same metronome judgment task from Experiment 1 to make within-individual comparisons for auditory and visual metronome conditions. As our 
primary interest was in investigating modality differences between auditory and visual beat and measure perception, and not in comparing bimodal versus unimodal perception, we eliminated the audiovisual metronome condition. Previous research has demonstrated IQ and musical training are positively correlated, but the direction of causality is unclear (Schellenberg, 2011). To control for non-training-related cognitive differences in task performance between musicians and non-musicians, we assessed general cognitive ability (IQ) in a separate task and used it as an individual differences control variable. Additionally, some individuals are better at rhythm perception than others, regardless of lack of musical training ("musical sleepers") while some individuals with musical training are poorer music perceivers than would be assumed ("sleeping musicians"; Law \& Zentner, 2012). As we wished to assess the effects of accumulated experience (music/dance training) on perception, we assessed basic rhythm perception abilities in a separate music aptitude task to independently assess both aptitude and experience.

\section{Method}

\section{Participants}

Thirty-two individuals (18 female) who did not participate in the previous experiment participated in Experiment 2. Participants were recruited from the Department of Psychology participant pool and the Department of Music at the University of Nevada, Las Vegas, and from the surrounding community. We recruited participants with a high amount of music training to be part of the sample, along with individuals from the general participant pool, resulting in a varied sample (see Table 1 for full demographic information). All participants reported normal hearing and normal or corrected-to-normal hearing, and spoke English, either having learned English as their first language $(n=25)$ or during childhood $\left(n=7, M_{\text {age learned }}=8.86\right.$ years, $S D=$ 
2.11 years, range: 5-12 years). One additional participant completed one experimental session but declined to return for the second session: their data are not included.

\section{Power Analysis.}

As in Experiment 1 we examined interactions between beat- or measure-level perception and metronome modality. In Experiment 1, we observed significant main effects of visual modality $(\gamma=0.47)$ and a significant interaction of beat and visual modality $(\gamma=-0.46)$. By changing metronome modality to a within-participants (level 1) variable and doubling the number of trials per person to 192 (96 trials/modality), we estimated that a minimum of 27 participants would be needed to reach a power of 0.8 to detect fixed effects, with minimum effect sizes of $d=0.20$ and effect size variances of $0.10\left(S D_{\text {effect size }}= \pm 0.30\right)$. Larger actual effect sizes and/or smaller variances required fewer participants to achieve adequate power (Raudenbush \& Liu, 2000; Spybrook et al., 2011). Our sample size of 32 participants with 192 trials per participant exceeded this threshold and was above the 30/30 rule suggested for adequate power in multilevel designs (Hox, 1995, 2010).

\section{Stimuli}

All stimuli were identical to those used in corresponding conditions of Experiment 1 . The visual metronomes were the "upright" style (Figure 3A).

\section{Measures}

Participants completed the Vocabulary and the Matrix Reasoning subtests of the Wechsler Abbreviated Scale of Intelligence, Second Edition (WASI-II; Wechsler \& Hsiao-Pin, 2011) as the measure of general cognitive ability (IQ). The Vocabulary sub-test measures verbal intelligence, and the Matrix Reasoning sub-test measures non-verbal intelligence. In the Vocabulary sub-test, participants are asked to define a set list of words. In the Matrix Reasoning 
sub-test, participants select the figure or image from a larger set that completes a larger incomplete pattern. Taken together, the two subtests yield an estimated IQ score. The WASI-II was administered verbally by the experimenter in English. Each sub-test took approximately 10 minutes per participant. Raw scores on the subtests were converted to age-normed $t$-scores, then summed and converted to IQ estimates.

To provide an objective measure of music perception skills beyond reporting musical training, participants completed the Gordon Advanced Measures of Music Audiation (AMMA; Gordon, 1986). We used a computerized version of the AMMA, which outputs a summed score, a melody score, and a rhythm score. We used raw rhythm scores for our analysis. Higher scores indicate better performance. Participants took approximately 15 minutes to complete the test.

\section{Procedure}

Experiment 2 took place over two experimental sessions lasting approximately 60 minutes each, with a minimum two-day gap between sessions $(M=3.44$ days, $S D=2.23$, range: $2-12$ ). The gap was meant to avoid any carryover effects from auditory to visual metronome modalities (as observed in Grahn et al., 2011 and McAuley \& Henry, 2010). Order of metronome modality was counterbalanced across participants. In a session, participants completed the metronome matching task first (either auditory-only or visual-only), and the assessments second (IQ testing in session 1, music perception testing in session 2).

The apparatus, experimental program, instructions, and procedure were identical to the visual (upright) and auditory metronome conditions in Experiment 1, with participants receiving 96 trials per metronome modality. Participants completed a demographic questionnaire at the end of session 2. All procedures were approved by the Institutional Review Board (IRB) of the 
University of Nevada, Las Vegas, and all participants were treated in an ethical manner consistent with the APA Code of Ethics.

\section{Data Analysis}

We used MLM to examine how fit ratings varied by metronome modality and beat- and measure-level matching, while controlling for factors within and between participants. We based the models in Experiment 2 on those in Experiment 1. The dependent variable in all models was participants' ratings of fit. First, we hypothesized we would replicate the auditory advantage for beat-level perception, as indicated by beat $\mathrm{x}$ modality, measure $\mathrm{x}$ modality, and beat $\mathrm{x}$ measure $\mathrm{x}$ modality interactions. Model 1 contained the within-subjects variables of beat (matching or mismatching), measure (matching or mismatching), modality (auditory or visual), tempo (slow, medium, fast), and notated meter (duple or triple), along with the interaction terms, and the between-subjects variables of music training, dance training, music listening per week, IQ, and AMMA rhythm raw score. We included random effects for beat-level asynchrony, measure-level asynchrony, and metronome modality to control for stimulus-level variability.

Second, we hypothesized that formal musical and/or dance training relates to differences in meter perception, either enhancing or decreasing sensitivity to given metrical levels or modalities. Using the within-subjects design for modality in Experiment 2, we again examined relationships between formal training (music or dance), sensitivity to metrical information, and metronome modality. We assessed these in Model 2 by adding cross-level interaction terms of beat $\mathrm{x}$ training, measure $\mathrm{x}$ training, and beat or measure $\mathrm{x}$ modality $\mathrm{x}$ training. Both models were compared to a baseline model (one-way random effects ANOVA).

All within-participant (Level 1) predictors were dichotomous variables, coded as in Experiment 1, with the addition of modality as dichotomous, with 0 for auditory and 1 for visual. 
All between-participant predictors (Level 2) were grand-mean centered and unstandardized. There were six total missing ratings of fit across the entire dataset $(<1 \%)$ : with one person missing four trials ( $2 \%$ of their total). We dropped the trials with missing responses from the analysis. One participant failed to report years of music training for the between-subjects factors (Level 2). We mean-replaced the missing value for years of music training. Models were run with the same parameters as Experiment 1, and all models presented successfully converged. The full datasheets and the R scripts used are available on the OSF project page.

\section{Results and Discussion}

Ratings of fit are presented in Figure 5, and MLM results are reported in Table 3. In the baseline (intercept-only) model, most of the variance in ratings was attributable to withinsubjects variance $(1.36,95 \% \mathrm{CI}: 1.31-1.41)$ and a small portion to between-subjects variance $(0.05,95 \%$ CI: $0.03-0.09)$. Approximately $96 \%$ of the variance was within-subjects (experimental manipulations) and $4 \%$ between-subjects (individual differences). 


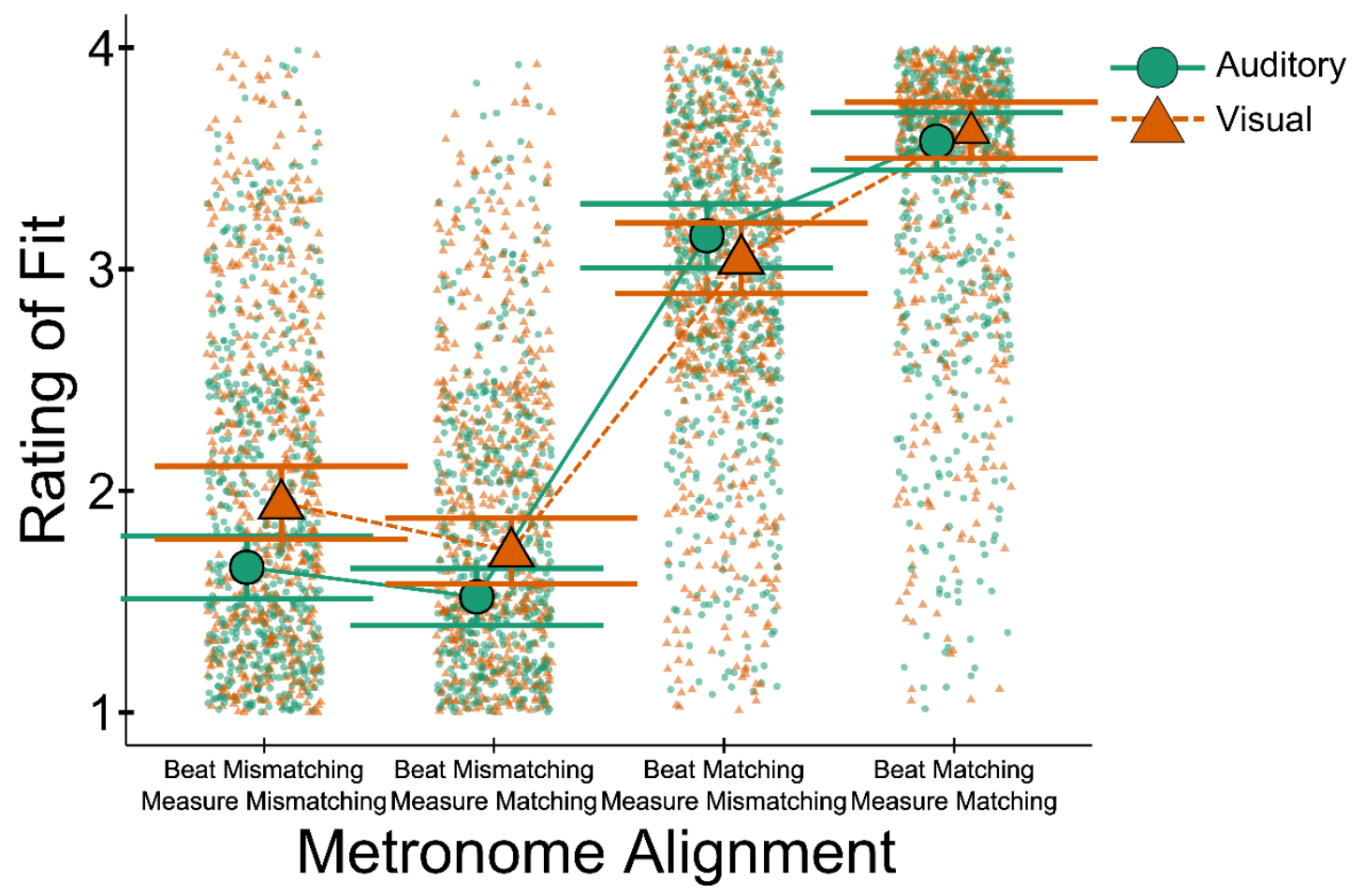

Figure 5. Ratings of fit between metronome and music by beat- and measure-level alignment. The responses ranged from 1-4 in integers: horizontal and vertical jitter and opacity changes have been added to the graph to aid in visualization. All responses ( $n=32$ participants, 192 responses each; approximately 6100 total responses) are plotted with shapes and color representing modality. All participants completed both auditory and visual conditions. Means and $95 \%$ confidence intervals for each metronome alignment and modality are plotted on top of the entire distribution of responses. Please see the online version of the article for the color version of this figure. 
Table 3. Results of Multilevel Models in Experiment 2 Investigating Differences in Ratings of Fit of Metronome to Music as a function of Within- and Between-Participant Factors

\begin{tabular}{|c|c|c|c|}
\hline Parameter & Baseline & Model 1 & Model 2 \\
\hline \multicolumn{4}{|c|}{ Fixed Effects } \\
\hline Rating of Fit (Intercept) & $2.532 * *$ & $1.810^{* *}$ & $1.784 * *$ \\
\hline \multicolumn{4}{|l|}{ Within-Subjects Factors (Level 1) } \\
\hline Beat & & $1.330^{* *}$ & $1.353 * *$ \\
\hline Measure & & $-0.139 *$ & $-0.123^{*}$ \\
\hline Slower Tempo & & $0.059^{*}$ & $0.056^{*}$ \\
\hline Faster Tempo & & $0.059 *$ & $0.062 * *$ \\
\hline Musical Meter & & $-0.121 * *$ & $-0.124 * *$ \\
\hline Modality & & $0.307 * *$ & $0.299 * *$ \\
\hline Beat $x$ Measure & & $0.515^{* *}$ & $0.520 * *$ \\
\hline Beat x Modality & & $-0.392 * *$ & $-0.392 * *$ \\
\hline Measure x Modality & & -0.084 & -0.084 \\
\hline Beat $x$ Measure x Modality & & $0.234 * *$ & $0.234 * *$ \\
\hline Beat $\mathrm{x}$ Music Training & & & 0.010 \\
\hline Measure x Music Training & & & $0.016 * *$ \\
\hline Beat $x$ Measure $x$ Music Training & & & 0.004 \\
\hline Beat $x$ Dance Training & & & $0.045^{*}$ \\
\hline Measure x Dance Training & & & -0.009 \\
\hline Beat $x$ Measure $x$ Dance Training & & & 0.015 \\
\hline Modality x Music Training & & & $-0.013 *$ \\
\hline Modality x Dance Training & & & 0.027 \\
\hline Beat x Modality x Music Training & & & 0.004 \\
\hline Measure x Modality x Music Training & & & $0.007 *$ \\
\hline Beat $x$ Modality x Dance Training & & & 0.018 \\
\hline Measure x Modality x Dance Training & & & $-0.054 * *$ \\
\hline \multicolumn{4}{|l|}{ Between-Subjects Factors (Level 2) } \\
\hline Music Training & & -0.002 & $-0.021 * *$ \\
\hline Dance Training & & 0.013 & -0.015 \\
\hline Music Listening & & 0.004 & 0.004 \\
\hline IQ Score & & -0.005 & -0.005 \\
\hline Rhythm Score & & $0.037 * *$ & $0.037 * *$ \\
\hline \multicolumn{4}{|c|}{ Random Effects } \\
\hline Within-Subjects (Residual) & $1.359^{* *}$ & $0.521^{* *}$ & $0.517 * *$ \\
\hline Between-Subjects (Intercept) & $0.049 * *$ & $0.107 * *$ & $0.090 * *$ \\
\hline Intraclass Correlation (ICC) & 0.035 & 0.170 & 0.148 \\
\hline
\end{tabular}

Note. All coefficients are unstandardized. Between-participants (Level 2) variables are grandmean centered. $* p<.05, * * p<.01$ 


\section{Hypothesis 1: Auditory Advantage for Beat Perception Within Individuals}

Model 1 tested the first hypothesis by examining the effects of modality (auditory or visual), beat, measure, and the interactions among beat, measure, and modality while controlling for music training, dance training, music listening, IQ $(M=105.12, S D=13.22$, Range: $78-135)$, and rhythm score $(M=29.13, S D=3.78$, Range: $20-37)$ as fixed effects, with random effects for beat-level asynchrony, measure-level asynchrony, and metronome modality.

As in Experiment 1, the beat $\mathrm{x}$ measure interaction was significant, with beat-level matching moderating the effect of measure-level matching. When the beat of the metronome matched the music, participants gave higher ratings to measure-matching $(3.52,95 \% \mathrm{CI}: 3.41-$ 3.69) than measure-mismatching metronomes $(3.14,95 \% \mathrm{CI}: 2.85-3.43, \omega=0.38,95 \% \mathrm{CI}$ : $0.27-0.48)$. When the beat of the metronome mismatched the music, participants gave lower ratings to measure-matching $(1.67,95 \% \mathrm{CI}: 1.56-1.78)$ than measure-mismatching metronomes (1.81, 95\% CI: $1.67-1.94, \omega=-0.14,95 \%$ CI: $-0.25--0.03)$. As in Experiment 1, the random effects of beat- and measure-level asynchrony were again significant $(p<.001)$. Both beat-level and measure-level asynchrony predicted lower ratings $\left(r_{\text {beat-level asynchrony,intercept }}=-.67 ; r_{\text {measure-level }}\right.$ asynchrony,intercept $=-.41)$, and this relationship was stronger for beat-level than measure-level asynchrony, as in Experiment 1.

The beat $\mathrm{x}$ modality interaction was statistically significant. Participants were more sensitive to beat in the auditory metronome condition $(\omega=1.33,95 \%$ CI: $1.17-1.49)$ than in the visual metronome condition $(\omega=0.94,95 \% \mathrm{CI}: 0.78-1.09)$. The measure $\mathrm{x}$ modality interaction was not significant $(\gamma=-0.084,95 \% \mathrm{CI}:-0.13--0.03 p=.106)$. Thus, as in Experiment 1 , we found an auditory advantage for beat but not for measure perception. 
The beat $\mathrm{x}$ measure $\mathrm{x}$ modality interaction was also statistically significant. When the beat of the metronome matched the music, participants were more sensitive to measure in visual than auditory metronome conditions ( $\left.\omega_{\text {difference }}=0.15, p=.01\right)$. When the beat of the metronome mismatched the music, ratings did not significantly differ by modality $\left(\omega_{\text {difference }}=-0.08, p=\right.$ .123). This result suggests that individuals might have better perception of multiple simultaneous metrical levels, or perhaps just slower levels, when the metronome is visual than when it is auditory, something we did not observe in Experiment 1.

In Model 1, the addition of the within-subjects predictors accounted for approximately $62 \%$ of the unexplained within-subjects variance relative to the unconstrained (baseline) model. The proportion of unexplained variance between-subjects increased to $17 \%$ and the proportion of unexplained within-subjects variance decreased to $83 \%$.

\section{Hypothesis 2: Training Relates to Enhanced Meter Perception}

In Model 2, we assessed the hypothesis that formal training would relate to enhanced sensitivity to beat- and measure-level information in auditory and visual metronomes. All previous significant fixed effects remained statistically significant (Table 3). The relevant interaction terms of beat $\mathrm{x}$ dance training, measure $\mathrm{x}$ modality $\mathrm{x}$ music training, and measure $\mathrm{x}$ modality $\mathrm{x}$ dance training were statistically significant.

The simple-slopes test of the beat $\mathrm{x}$ dance training interaction demonstrated that participants with more dance training ( $+1 S D$ above the mean; 3.89 total years of training) were more sensitive to beat-level information $(\omega=1.48,95 \%$ CI: $1.29-1.63)$ than individuals without dance training $(\omega=1.31,95 \% \mathrm{CI}: 1.16-1.46)$. We did not find a significant relationship between beat and music training. 
As in Experiment 1, we observed a measure $\mathrm{x}$ modality $\mathrm{x}$ music training interaction. Musicians (+1SD) gave similar ratings of fit to measure-matching and mismatching metronomes in both auditory and visual conditions $\left(\omega_{\text {auditorymusician }}=0.07, \omega_{\text {visualmusician }}=0.08, \omega_{\text {differencemusician }}=\right.$ $0.01 ; p>.5)$. Non-musicians gave lower ratings to measure-matching than mismatching metronomes, and this effect was larger for visual than auditory metronomes $\left(\omega_{\text {auditorynonmusician }}=\right.$ $\left.0.29, \omega_{\text {visualnonmusician }}=-0.44, \omega_{\text {differencenonmusician }}=-0.16 ; p=0.018\right)$. As in Experiment 1 , musicians may have been more able to disregard beat-level asynchrony and attend to measure-level information in both modalities.

Conversely, both dancers $(+1 S D)$ and non-dancers gave lower ratings to measurematching metronomes than measure-mismatching metronomes, but for dancers this pattern was larger in the visual condition than in the auditory condition $\left(\omega_{\text {auditory }}=-0.15, \omega_{\text {visual }}=-0.39\right.$, $\left.\omega_{\text {difference }}=-0.24 ; p=0.001\right)$, whereas for non-dancers the effect of measure did not differ by modality $\left(\omega_{\text {auditory }}=-0.12, \omega_{\text {visual }}=-0.15, \omega_{\text {difference }}=0.03 ; p>.5\right)$.

The random effects in Model 2 remained similar to Model 1, but with a decreased effect for measure-level asynchrony $\left(r_{\text {beat asynchrony,intercept }}=-.62 ; r_{\text {measure asynchrony,intercept }}=-.26\right)$. In Model 2 , the additional cross-level interaction terms accounted for $9 \%$ of the previously unexplained between-participants variance from Model 1. After fitting Model 2 to the data, approximately $14 \%$ of the remaining unexplained variance in ratings was between-subjects and $86 \%$ was within-subjects.

\section{Summary}

As in Experiment 1, adults gave the highest ratings of fit to metronomes that matched the music at two metrical levels in both modalities. We again found evidence for an auditory 
advantage for beat perception, and in this experiment we observed a slight advantage for perceiving two levels of meter simultaneously in visual metronomes.

We found significant three-way interactions involving music and dance training, even while controlling for overall rhythm perception ability through AMMA rhythm scores. Music training was associated with similar sensitivity to the measure-level information in both modalities compared to non-musicians, as we observed in Experiment 1. However, unlike Experiment 1, where dance training did not predict sensitivity to beat, dancers in Experiment 2 were more sensitive than non-dancers to beat-level information in both modalities. We also found in Experiment 2 that dancers and non-dancers alike gave lower ratings to measurematching than measure-mismatching metronomes. If we assume that this tendency arises from greater attention to beat-level synchrony as we proposed above, then both Experiment 1 and 2 may suggest evidence that dancers, along with non-musicians, are more attuned to beat-level synchrony than to measure-level synchrony. However, levels of dance training remained low in Experiment $2(M=0.98$ years, $S D=2.91)$ : the true effect of dance training may be small, and thus it may require larger amounts of training to see consistent effects. In both cases, future studies using this paradigm but with larger sample sizes administered to a wide range of individuals, or specifically targeting individuals with formal training, may better address the question of the relationships between training, modality, and beat- and measure-level perception.

We successfully replicated the findings of the auditory advantage for beat- but not measure-level perception and found significant relationships between formal training and perception in both a between-subjects and within-subjects design. Experiments 1 and 2 established that a clear auditory advantage exists for beat, but not measure perception. However, it is unclear whether the auditory advantage for beat is relatively stable throughout childhood or 
if it increases with age and the attendant accumulation of auditory experience. As reviewed above, prior research suggests that visual timing lags behind auditory timing during development (Innes-Brown et al., 2011; Nava \& Pavani, 2013; Parker \& Robinson, 2018), while other evidence suggests that more complex metrical and beat perception depends on life-long experience listening to music (Braun Janzen et al., 2014; Drake, Jones, et al., 2000; Einarson \& Trainor, 2016; McAuley et al., 2006; Mu et al., 2017; Nave-Blodgett et al., 2021; Puyjarinet et al., 2017; Thompson et al., 2015). If the auditory modality is inherently superior for perceiving beat, children should display a robust auditory advantage even in early childhood that remains relatively consistent with age. Alternatively, if experience is a large component of the auditory advantage for beat and meter processing because perceiving music in our culture is predominantly an auditory experience, we might expect to see an auditory advantage for beat that increases with age.

\section{Experiment 3}

In Experiment 3, we tested children's beat and meter perception with auditory and visual metronomes. To control for individual differences in cognitive abilities, we measured children's IQ using the same assessments as in Experiment 2.

\section{Method}

\section{Participants}

Ninety-two children (52 female), ranging from 5 to 10 years of age, were recruited to participate in a visual metronome perception task. They were distributed across three contiguous age groups of roughly equal size. Families from the Las Vegas community with children of eligible ages volunteered to bring their children in to participate in response to fliers and advertisements. We did not specifically recruit children with or without dance or music training, 
but there was variation in training across the age groups (see Table 4 for full demographic information). The caregivers of all children reported that the children had no known uncorrected hearing or visual impairments and were either in good health or had no more than a minor cold or illness on the day of testing. We then compared the data from this sample of 92 children who completed the task with visual metronomes to the data from a sample of 93 children, ages 5-10, who completed a similar task with auditory metronomes around the same time (Nave-Blodgett et al., 2021).

All testing procedures for both data sets were conducted in English. All children received a toy and a t-shirt after the testing session as a thank-you for their participation. An additional 12 children began the task but were excluded from the final sample because they were unwilling to complete the experiment $(n=8)$, or they failed to follow instructions (i.e. gave the same response throughout the entire experiment and/or admitted they ignored instructions, $n=4$ ). 
Table 4. Experiment 3 Participant Demographic Information

\begin{tabular}{|c|c|c|c|c|c|c|c|}
\hline \multirow[b]{2}{*}{ Characteristic } & \multirow{2}{*}{$\begin{array}{l}\text { Full Sample } \\
\text { (all } \\
\text { participants) }\end{array}$} & \multicolumn{2}{|c|}{ 5-6 year-olds } & \multicolumn{2}{|c|}{$7-8$ year-olds } & \multicolumn{2}{|c|}{$9-10$ year-olds } \\
\hline & & Auditory & Visual & Auditory & Visual & Auditory & Visual \\
\hline$n$ ( $n$ Females $)$ & $185(103)$ & $30(14)$ & $33(26)$ & $31(15)$ & $31(15)$ & $31(22)$ & $29(11)$ \\
\hline Age in Years & $7.37( \pm 1.74)$ & $5.27( \pm 0.45)$ & $5.39( \pm 0.50)$ & $7.35( \pm 0.49)$ & $7.58( \pm 0.50)$ & $9.48( \pm 0.51)$ & $9.34( \pm 0.48)$ \\
\hline Age in Months & $\begin{array}{c}94.20 \\
( \pm 20.90)\end{array}$ & $70( \pm 5.57)$ & $\begin{array}{c}69.97 \\
( \pm 5.87)\end{array}$ & $95( \pm 7.43)$ & $\begin{array}{c}95.52 \\
( \pm 6.55)\end{array}$ & $\begin{array}{l}119.84 \\
( \pm 6.69)\end{array}$ & $\begin{array}{l}117.14 \\
( \pm 7.14)\end{array}$ \\
\hline Age Range in Months & $61-130$ & $61-82$ & $61-81$ & $85-106$ & $84-107$ & $108-130$ & $108-129$ \\
\hline$n$ Hispanic (\%) & $42(23 \%)$ & $5(17 \%)$ & $4(12 \%)$ & $9(29 \%)$ & $7(23 \%)$ & $10(32 \%)$ & $7(24 \%)$ \\
\hline$n$ bilingual (\%) & $43(23 \%)$ & $7(23 \%)$ & $4(12 \%)$ & $9(29 \%)$ & $10(32 \%)$ & $6(19 \%)$ & $7(24 \%)$ \\
\hline $\begin{array}{l}n \text { Mother with } 4 \text {-year } \\
\text { college education or } \\
\text { higher }(\%)\end{array}$ & $105(57 \%)$ & $16(53 \%)$ & $20(61 \%)$ & $15(48 \%)$ & $22(71 \%)$ & $15(48 \%)$ & $17(59 \%)$ \\
\hline $\begin{array}{l}n \text { Participants with Music } \\
\text { Training (\%) }\end{array}$ & $59(32 \%)$ & $7(23 \%)$ & $6(18 \%)$ & $9(29 \%)$ & $11(37 \%)$ & $12(39 \%)$ & $14(48 \%)$ \\
\hline Years Music Training & $0.61( \pm 1.18)$ & $0.24( \pm 0.50)$ & $0.27( \pm 0.69)$ & $0.64( \pm 1.29)$ & $0.53( \pm 0.88)$ & $0.96( \pm 1.59)$ & $1.03( \pm 1.54)$ \\
\hline Range of Music Training & $0.10-5.50$ & $0.50-2.00$ & $0.50-3.00$ & $0.33-5.00$ & $0.50-3.00$ & $0.20-5.50$ & $0.10-5.00$ \\
\hline $\begin{array}{l}n \text { Participants with Dance } \\
\text { Training }(\%)\end{array}$ & $85(46 \%)$ & $11(37 \%)$ & $17(52 \%)$ & $11(35 \%)$ & $15(48 \%)$ & $20(65 \%)$ & $11(38 \%)$ \\
\hline Years Dance Training & $0.93( \pm 1.53)$ & $0.46( \pm 0.92)$ & $0.70( \pm 1.00)$ & $0.80( \pm 1.50)$ & $0.93( \pm 1.31)$ & $1.73( \pm 2.13)$ & $0.94( \pm 1.80)$ \\
\hline Range of Dance Training & $0.05-8.00$ & $0.05-3.50$ & $0.25-4.00$ & $0.15-6.00$ & $0.25-5.00$ & $0.15-8.00$ & $0.25-6.00$ \\
\hline $\begin{array}{l}\text { Hours Music Listening/ } \\
\text { Week }\end{array}$ & $6.99( \pm 6.84)$ & $7.83( \pm 6.45)$ & $5.42( \pm 4.61)$ & $7.69( \pm 7.98)$ & $6.64( \pm 5.22)$ & $8.96( \pm 9.99)$ & $5.46( \pm 5.35)$ \\
\hline WASI-II FSIQ-2 & $\begin{array}{c}104.16 \\
( \pm 15.59)\end{array}$ & $\begin{array}{c}97.59 \\
( \pm 13.37)\end{array}$ & $\begin{array}{c}100.58 \\
( \pm 13.39)\end{array}$ & $\begin{array}{c}104.72 \\
( \pm 15.05)\end{array}$ & $\begin{array}{c}109.65 \\
( \pm 19.65)\end{array}$ & $\begin{array}{c}102.65 \\
( \pm 11.66)\end{array}$ & $\begin{array}{c}109.72 \\
( \pm 16.39)\end{array}$ \\
\hline
\end{tabular}

Note. All values are means and standard deviations unless otherwise specified. Years music training and years dance training include all participants. Ranges of music and dance training contain only participants with relevant training. All values based on caregiver report of child information. FSIQ-2: full-scale IQ estimate based on two WASI-II subtests (Matrix Reasoning and Vocabulary). 


\section{Power Analysis.}

Our primary interest was to examine relationships between children's age, metronome modality, and their perception of meter, which could emerge as two-way (e.g., beat x measure, beat $\mathrm{x}$ modality, beat x age, etc.) or three-way (e.g., beat x measure x age, beat x modality x age, etc.) cross-level (within-between) interactions. In adults in Experiments 1 and 2, the beat $x$ modality interaction ranged between $|\gamma|=0.39-0.60$. A previous investigation of children's and adolescents' synchronization to visual and auditory metronomes found significant main effects and interactions of age and modality ranging from $\eta_{p}^{2}$ of .17 to .29 (Mu et al., 2017), roughly equivalent to $d=0.8-1.2$ (Fritz et al., 2012). Based on these results, we estimated our effect sizes conservatively. For this experiment, metronome modality was a between-subjects variable. To detect fixed effects with a minimum effect size of $d=0.15$ with a power of 0.8 , assuming an ICC of 0.10 and an $r^{2}=0.05$ of age (conservative), we estimated we required a minimum of 165 total participants with 48 trials per child (Raudenbush \& Liu, 2000; Spybrook et al., 2011). Higher effect sizes (of fixed effects and/or of age) required fewer participants to achieve power of .80. Our sample size for the analysis of 185 participants with 48 trials each ensured adequate power and was above the 30/30 minimum for multilevel designs (Hox, 1995, 2010).

\section{Stimuli and Measures}

Stimuli were identical to Experiment 2. Because participant fatigue and retention is often a concern with child participants, we shortened the study: children experienced 48 musical excerpt/metronome pairings (half what adults experienced). Because Experiments 1 and 2 revealed an auditory advantage in both between- and within-subjects designs, we chose to employ a between-participants design and present only one metronome modality per participant. 
We performed the same brief IQ assessment for all participants in Experiment 3 as in Experiment 2: the two-subscale version of the WASI-II (Wechsler \& Hsiao-Pin, 2011). We converted raw scores to standardized scores and IQ estimates based on the published age norms. However, because the WASI-II is only normed to age 6, all 5-year-old participants' scores were converted using the 6 years/0 months age norms.

\section{Procedure}

All participants were tested individually on an iMac computer (Apple, Inc., Cupertino, CA) booted into Windows 7 (Microsoft Corporation, Seattle, WA) running Presentation Software. Participants listened to the musical stimuli over child-sized headphones (Kidz Gear, El Dorado Hills, CA) and entered their ratings of fit on a Cedrus RB-830 response pad (Cedrus Corporation, San Pedro, CA). The buttons were equipped with different color key-caps (red, yellow, green, and blue) that matched the visual depiction of the ratings in the program.

We added storylines to the task to make the experiment more engaging for younger participants and to encourage completion. For auditory metronomes, the experimenter told each participant they were helping judge auditions of drummers seeking to join a musical band made up of bugs (insects). The experimenter read aloud the text displayed on the computer screen: "Bugsy [the main character] needs your help to judge how good the performances are. He is asking you to be an audition judge, and let him know how good the drummers play along with his songs. [...] To be an audition judge, you will listen to the song and the drummer together, and then tell Bugsy how well the drummer played along with Bugsy’s song."

For visual metronomes, the experimenter introduced each participant to the same main bug character and another bug who had made "musical clocks" that would tick along and match the music that the main bug "sang". The participant's job was to help "judge" how well the 
clocks matched the music that the bugs sang. The experimenter read aloud the text displayed on the computer screen: "Bugsy [the main character] needs your help to figure out if the clocks Sam [the other bug character] made tick along with the music from Bugsy. [...] Bugsy would like you to tell him how well the clocks ticked along with Bugsy's music. [...] Let's listen to a clock and Bugsy's music, and we'll tell Bugsy how well that clock matched Bugsy's music.”

After each experimental trial, children were prompted to rate the match between the metronome (either "bug drummer" or "clock") and the music, with the text "How well did the [bug drummer/clock] match Bugsy's song?" The children used four colored buttons (red, yellow, green, and blue) on the response pad to enter their ratings. Each color corresponded to a rating from "1" to "4", as in Experiments 1 and 2. This scale was additionally illustrated with colormatched "thumbs-up" or "thumbs-down" icons visible on the computer screen: red (1) was shown with "two thumbs down," yellow (2) with "one thumb down," green (3) with "one thumb up," and blue (4) with "two thumbs up." This explanation of the scale (colors and images) was visible every time the participant entered a rating of fit between a musical excerpt and metronome pair for both metronome modalities, and the experimenter reminded the participant as often as needed.

The test trials were divided into three blocks of 16 trials each, with metronome/music pairing order randomized within block and between participants. Children also completed six demonstration trials and six practice trials (the music/metronome pairings were the same in the demonstration and practice trials), double the number as adults: two fully-matching, two beatmatching measure-mismatching, and two fully-mismatching metronome/music pairs. All participants were given the option to repeat the practice trials if the participant did not understand the task by the end of the practice. 
After completing the metronome judgment task, the experimenter or a research assistant administered both subtests of the WASI-II. Participants generally took 10-15 minutes to complete the two subtests. WASI scores were not available for 5 participants, due to experimenter error $(n=3)$ or refusal to participate/answer questions $(n=2)$.

While the participant completed the experiment, the caregiver(s)/parent(s) of the participant completed a demographic questionnaire assessing the participants' developmental, language, music, and dance history. All procedures were approved by the Institutional Review Board (IRB) of the University of Nevada, Las Vegas, and all participants were treated in an ethical manner consistent with the APA Code of Ethics.

\section{Data Analysis}

We used MLM to examine how children's fit ratings varied by beat- and measure-level matching, metronome modality, formal training, and participant age while controlling for other within- and between-participant factors. We had three hypotheses: Our first hypothesis was that older children would be more sensitive to meter than younger children, regardless of metronome modality, which could give rise to beat $\mathrm{x}$ age, measure $\mathrm{x}$ age, and/or beat $\mathrm{x}$ measure $\mathrm{x}$ age interactions. Second, we expected to observe an auditory advantage for meter perception among children, but that it might vary as a function of age, which could give rise to beat x modality, measure x modality, age x modality, and beat/measure x age x modality interactions. In Model 1, we added the above interaction terms along with the within-subjects variables of beat, measure, tempo, musical meter, beat x measure, and between-subjects variables of metronome modality (auditory or visual), age (in months), music training, dance training, music listening, and IQ (as a covariate). As in previous experiments, we included beat-level and measure-level asynchrony between the metronome and music as random effects. Our third hypothesis was that formal 
music or dance training would relate to sensitivity to beat- and/or measure-level information, as it did in adults (Experiments 1 and 2). We were again unsure if expertise would moderate perception differently based on metronome modality, so in Model 2 we added the beat x training (music/dance), measure $\mathrm{x}$ training, and beat $\mathrm{x}$ measure $\mathrm{x}$ training cross-level interactions into the model.

Within-subjects (Level 1) predictors were dichotomous and used the same coding scheme as Experiment 2. Between-subjects (Level 2) predictors beyond the dichotomous metronome modality ( 0 for auditory, 1 for visual) were grand-mean centered, but not standardized. There were no missing trial responses, and no missing values for within-subject (Level 1) factors. Most between-subjects (Level 2) data were present, but we did not have values for hours of music listening for twenty-five participants and IQ score estimates for five participants. We meansubstituted values for the missing Level 2 predictors. The same statistical analysis software and parameters were used as in previous experiments. Model 1 successfully converged, but Model 2 did not; code for models and datasheets are available on the OSF page.

\section{Results and Discussion}

Ratings of fit are presented in Figure 6, separated by age group and metronome modality. MLM results are reported in Table 5. In the baseline model, most of the variance in ratings of fit was within-subjects $(1.12,95 \%$ CI: $1.09-1.16)$, and a small portion between-subjects $(0.08$, 95\% CI: $0.06-0.10$ ). Approximately $93 \%$ was within-subjects (experimental), and $7 \%$ to between-subjects (individual differences). 

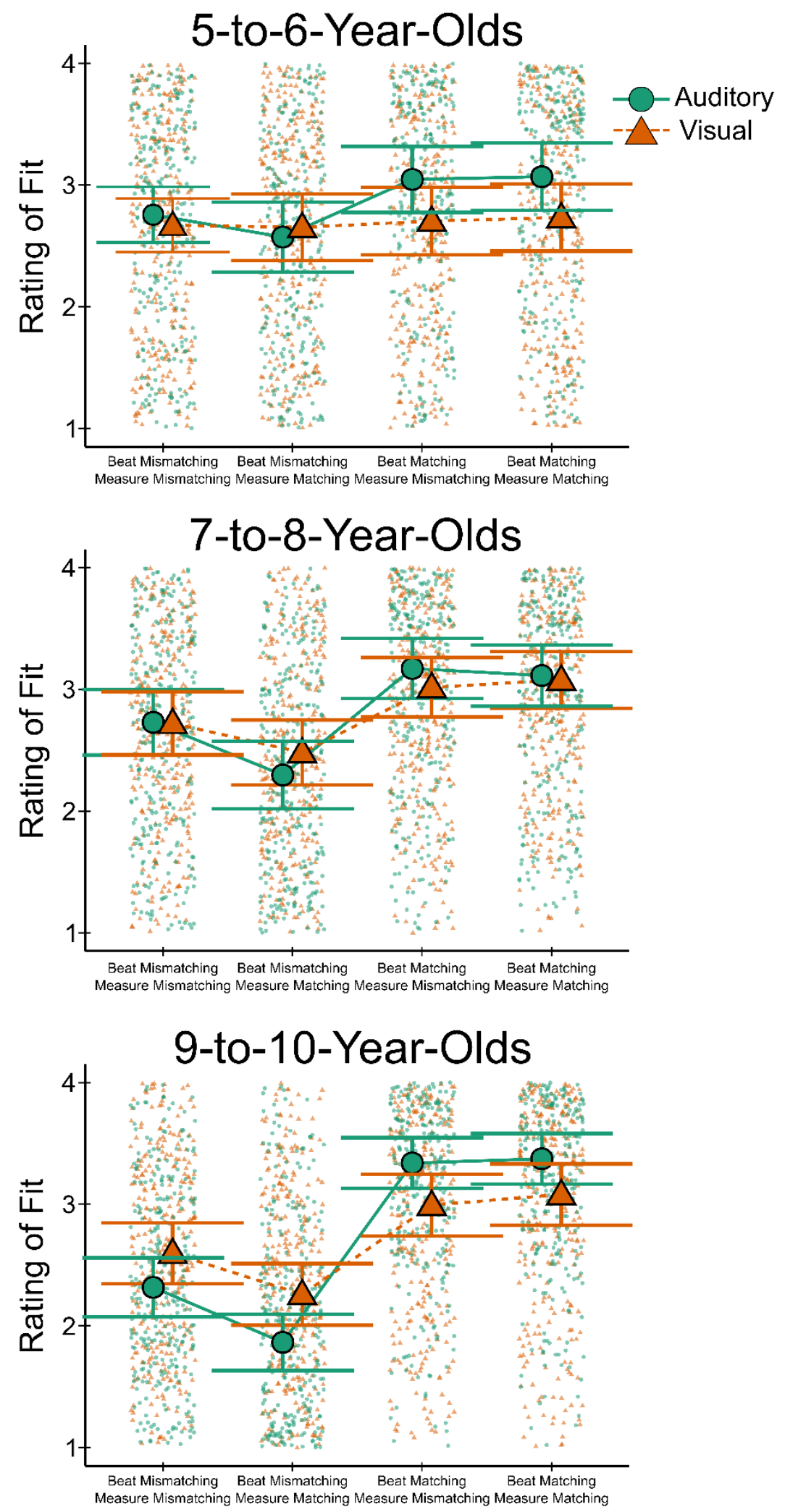
Figure 6. Ratings of fit between metronome and music by beat- and measure-level, separated by age group. All responses are shown, along with the means and 95\% confidence interval of each age group and metronome modality. In the individual responses, modality is indicated by color and shape. Responses could range from 1-4 in whole numbers: horizontal and vertical jitter has been added to the placement of responses to facilitate visualization. 5-6-year-olds, auditory metronomes $(n=30)$; 7-8-year-olds, auditory metronomes $(n=31)$; 9-10-year-olds, auditory metronomes $(n=31)$; 5-6-year-olds, visual metronomes $(n=33)$; 7-8-year-olds, visual metronomes $(n=31)$; 9-10-year-olds, visual metronomes $(n=29)$. Please see the online version of this article for the color version of this figure. 
Table 5. Results of Multilevel Models in Experiment 3 Investigating Differences in Ratings of Fit of Metronome to Music as a function of Within-and Between-Participant Factors

\begin{tabular}{|c|c|c|c|}
\hline Parameter & Baseline & Model 1 & Model 2 \\
\hline \multicolumn{4}{|c|}{ Fixed Effects } \\
\hline Rating of Fit (Intercept) & $2.776^{* *}$ & $2.634 * *$ & $2.633 * *$ \\
\hline \multicolumn{4}{|l|}{ Within-Subjects Factors (Level 1) } \\
\hline Beat & & $0.568^{* *}$ & $0.567 * *$ \\
\hline Measure & & $-0.352 * *$ & $-0.351 * *$ \\
\hline Slower Tempo & & $0.065^{*}$ & $0.065^{*}$ \\
\hline Faster Tempo & & -0.010 & -0.010 \\
\hline Musical Meter & & $-0.071 * *$ & $-0.071 * *$ \\
\hline Beat $x$ Measure & & $0.349^{* *}$ & $0.349 * *$ \\
\hline Beat x Modality & & $-0.318 * *$ & $-0.317 * *$ \\
\hline Measure x Modality & & $0.147^{*}$ & $0.147^{*}$ \\
\hline Beat x Measure x Modality & & -0.088 & -0.088 \\
\hline Beat x Age & & $0.016^{* *}$ & $0.014 * *$ \\
\hline Measure x Age & & $-0.006 * *$ & $-0.005^{* *}$ \\
\hline Beat $x$ Measure $x$ Age & & $0.007 * *$ & $0.007 * *$ \\
\hline Modality x Age & & $0.009 * *$ & $0.007 *$ \\
\hline Beat $x$ Modality x Age & & $-0.007 *$ & -0.006 \\
\hline Measure x Modality x Age & & -0.001 & 0.000 \\
\hline Beat $\mathrm{x}$ Music Training & & & $0.112^{*}$ \\
\hline Measure x Music Training & & & 0.008 \\
\hline Modality x Music Training & & & 0.096 \\
\hline Beat $x$ Dance Training & & & 0.031 \\
\hline Measure $\mathrm{x}$ Dance Training & & & -0.013 \\
\hline Modality x Dance Training & & & 0.008 \\
\hline Beat x Modality x Music Training & & & -0.041 \\
\hline Measure x Modality x Music Training & & & -0.047 \\
\hline Beat x Modality x Dance Training & & & -0.022 \\
\hline Measure x Modality x Dance Training & & & 0.021 \\
\hline \multicolumn{4}{|l|}{ Between-Subjects Factors (Level 2) } \\
\hline Modality & & 0.041 & 0.042 \\
\hline Age (in months) & & -0.011 & $-0.009 * *$ \\
\hline Music Training & & 0.028 & -0.058 \\
\hline Dance Training & & -0.025 & -0.039 \\
\hline Music Listening & & 0.001 & 0.002 \\
\hline IQ Score & & 0.002 & 0.002 \\
\hline \multicolumn{4}{|c|}{ Random Effects } \\
\hline Within-Subjects (Residual) & $1.125^{* *}$ & $0.933^{* *}$ & $0.933 * *$ \\
\hline Between-Subjects (Intercept) & $0.080^{* *}$ & $0.117 * *$ & $0.116^{* *}$ \\
\hline Intraclass Correlation (ICC) & 0.066 & 0.111 & 0.110 \\
\hline
\end{tabular}


Note. All coefficients are unstandardized. Between-participants (Level 2) variables are grandmean centered (except for modality, which was dichotomous). Complete covariance matrix available on OSF as supplemental material. $* p<.05, * * p<.01$

\section{Hypothesis 1: Age and Modality Relate to Differences in Meter Perception}

Model 1 tested the hypothesis that children's perception of beat- and measure-level information varies depending on age and metronome modality. Like adults, children gave beatmatching metronomes higher ratings than beat-mismatching metronomes $(\gamma=0.57,95 \% \mathrm{CI}: 0.51$ - 0.62), and gave measure-matching metronomes lower ratings than measure-mismatching metronomes $(\gamma=-0.35,95 \%$ CI: $-0.39--0.31)$. The effect of beat-level matching (higher ratings) was smaller than in adults, and the effect of measure-level matching (lower ratings) was slightly larger than observed in adults.

The beat $\mathrm{x}$ measure interaction was significant. A simple-slopes test demonstrated that when the beat of the metronome matched the music, children gave equivalent ratings to measurematching $(3.19,95 \% \mathrm{CI}: 3.11-3.28)$ and measure-mismatching metronomes $(3.20,95 \% \mathrm{CI}$ : $3.10-3.30)$, controlling for all other factors $(\omega=-0.01,95 \% \mathrm{CI}:-0.09-0.09)$. When the beat of the metronome mismatched the music, children gave lower ratings to measure-matching metronomes $(2.28,95 \% \mathrm{CI}: 2.19-2.37)$ than measure-mismatching metronomes $(2.63,95 \% \mathrm{CI}$ : $2.54-2.73)$, when controlling for other factors $(\omega=-0.35,95 \% \mathrm{CI}:-0.44--0.26)$. These results suggest that unlike in adults, where beat-level matching moderated ratings of measure-level matching, in children, beat-level matching was the only aspect of the metronome they responded to.

Supporting this conclusion, the random effect of beat-level asynchrony was statistically significant $(p<.001)$, but the effect of measure-level asynchrony was not $(p=.311)$. There was 
again a significant negative relationship between beat-level asynchrony between the metronome

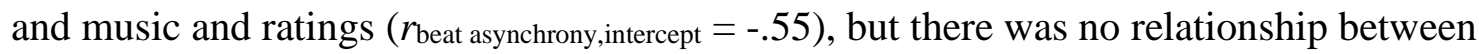

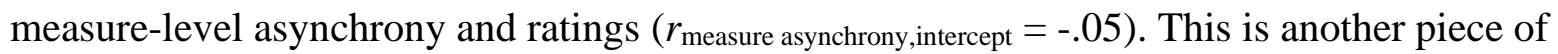
evidence strongly suggesting that children were not sensitive to measure-level information in the metronome and music, as the measure-level asynchrony between the metronome and music had no consistent relationship with ratings in children, unlike in adults.

The effect of beat and measure on ratings varied by age. A simple-slopes test of the beat $\mathrm{x}$ age interaction found that sensitivity to beat increased with age. While all age groups gave higher ratings to beat-matching than beat-mismatching metronomes, the $9-10$-year-olds $(\omega=$ 0.95, 95\% CI: $0.79-1.11)$ were more sensitive to beat than the 7-8-year-olds $(\omega=0.58,95 \%$ CI: $0.47-0.69)$, and the 5-6-year-olds were least sensitive to beat $(\omega=0.18,95 \%$ CI: $0.01-0.34)$. This strongly supports the idea that beat perception increases with age. The measure $\mathrm{x}$ age interaction was opposite: each successive age group gave increasingly lower ratings to measurematching metronomes compared to younger children $(5-6$ years: $\omega=-0.21,95 \% \mathrm{CI}:-0.33--$ 0.08, 7-8 years: $\omega=-0.36,95 \%$ CI: $-0.44--0.27,9-10$ years: $\omega=-0.49,95 \%$ CI: $-0.62--0.37)$. Prior work has demonstrated that given metronomes containing only measure-level ticks, children in the age ranges tested here do not readily match the measure-level information to the music in these conditions, while adults do (Nave-Blodgett et al., 2021). Taken with the lack of a significant random effect for measure-level asynchrony in this experiment, this supports our interpretation that the effect of measure observed here instead reflects children's beat perception.

The significant beat $\mathrm{x}$ measure $\mathrm{x}$ age interaction indicates that with increasing age, children's ratings become more differentiated by metronome alignment, except for the persistent lack of difference for measure-level information for beat-matching metronomes. When the beat 
of the metronome did not match the music, each successive age group gave increasingly lower ratings to measure-matching metronomes (all comparisons $p<.01 ; \omega_{5-6-Y e a r-O l d s ~}=-0.22, \omega_{7-8 \text {-Year- }}$ Olds $=-0.36, \omega 9-10-$ Year-Olds $=-0.49)$. When the metronome matched the beat of the music, all age groups gave the same ratings to measure-matching and measure-mismatching metronomes $\left(\omega_{5-6-}\right.$ Year-Olds $\left.=-0.04, \omega_{7-8-\text { Year-Olds }}=-0.01, \omega_{9-10-\text { Year-Olds }}=0.03\right)$. This suggests that children's ratings in this task are driven solely by beat-level match or mismatch, and are not influenced by measurelevel information.

\section{Hypothesis 2: The Auditory Advantage is Present in Childhood}

The interactions between beat $\mathrm{x}$ modality, measure $\mathrm{x}$ modality, and beat $\mathrm{x}$ age $\mathrm{x}$ modality were significant. The simple-slopes test of beat x modality revealed greater sensitivity to the beat in the auditory condition $(\omega=0.57,95 \% \mathrm{CI}: 0.46-0.68)$ than in the visual condition $(\omega=0.25$, 95\% CI: $0.14-0.36)$, confirming the presence of an auditory advantage for beat perception in childhood. For the measure x modality interaction, ratings of measure-matching metronomes were lower in the auditory $(\omega=-0.35,95 \% \mathrm{CI}:-0.44--0.26)$ than visual $(\omega=-0.21,95 \% \mathrm{CI}$ : $0.29--0.12$ ) metronome condition. As previously discussed, if we assume this effect is driven by sensitivity to beat-level information, the result further indicates an auditory advantage for beat.

There was also a significant beat $\mathrm{x}$ modality $\mathrm{x}$ age interaction. The size of the effect of beat grew with increasing age, but it was not uniform across modalities. When the beat of the metronome matched the music, the auditory condition received higher ratings than the visual condition at all ages studied. However, the difference in ratings between beat-matching auditory and visual metronomes grew smaller with increasing age $\left(\omega 5\right.$-6-Year-Olds $=-0.32, \omega_{7-8}$-Year-Olds $=$ 0.28, $\omega_{9-10-Y e a r-O l d s}=-0.23$ ), suggesting that visual beat (matching) perception increased with age. 
When the beat of the metronome mismatched the music, younger children gave similar ratings to auditory and visual metronomes, but older children gave lower ratings to auditory compared to visual metronomes $\left(\omega_{5-6-\text { Year-Olds }}=-0.18, \omega_{7-8 \text {-Year-Olds }}=0.04, \omega_{9-10-\text { Year-Olds }}=0.26\right)$. This suggests that with increasing age, children were more sensitive to the beat-level alignment of auditory metronomes but not visual metronomes. The corresponding interaction of modality, age, and measure was not significant.

In Model 1, the addition of the within-subjects predictors accounted for approximately $17 \%$ of the unexplained within-subjects variance relative to the baseline model. The proportion of between-participants variability increased to $11 \%$ and the proportion of within-participants variability decreased to $89 \%$.

\section{Hypothesis 3: Training Relates to Beat and Measure Perception in Children}

In Model 2, we assessed the hypothesis that music or dance training interacts with children's beat and/or measure perception, as it did in adults. We added the interaction terms among beat, measure, modality, and formal music and dance training. This model failed to converge successfully, thus changes in significance should be interpreted cautiously. All previous significant effects remained statistically significant (Table 5), except for the beat $\mathrm{x}$ modality $\mathrm{x}$ age interaction, which decreased slightly (Model 1: $\gamma=-0.007$, Model 2: $\gamma=-0.006$ ) and thus did not retain statistical significance $(p=.10)$. The only additional term in Model 2 to reach statistical significance was beat $\mathrm{x}$ music training.

Children with more music training (one standard deviation above the mean; 1.72 years total) were more sensitive to beat $(\omega=0.70,95 \% \mathrm{CI}: 0.55-0.85)$ than children with no music training $(\omega=0.50,95 \% \mathrm{CI}: 0.38-0.62)$, controlling for other factors. 
None of the other interaction terms added in Model 2 were statistically significant. The random effects remained the same as in Model 1. As Model 2 failed to converge, it did not account for additional unexplained between-subjects variance compared to Model 1.

\section{Summary}

Children's perception of meter was moderated by age and metronome modality. Older children were more sensitive to beat than younger children, and all age groups were more sensitive to beat in auditory than visual metronome conditions. Unlike adults, children did not demonstrate sensitivity to the measure-level or two levels of meter simultaneously with either auditory or visual metronomes at any age tested. We observed an auditory advantage for beat perception that was present at the earliest ages studied, and found evidence of a trend for the auditory advantage to increase with age. Further experimentation with younger and older cohorts of children could more clearly indicate if there is indeed an auditory advantage that increases with age. In children, music training related to enhanced sensitivity to beat-level information.

\section{General Discussion}

The present series of experiments was motivated by two main goals: first, to determine if there is an auditory advantage for matching a metronome with multiple levels of meter to complex music excerpts, and second, to understand the developmental trajectory of the auditory advantage. We paired rich, complex excerpts of multi-instrument music with auditory and visual metronomes and asked adults and children to rate the fit of the metronomes to the music. First, we consistently found an auditory advantage for perceiving the beat across all three experiments. Interestingly, we found no evidence of an auditory advantage for the measure level. Second, we found that the auditory advantage for beat perception was robust during childhood and it showed some evidence of increasing with age. Additionally, formal music training was related to 
enhanced auditory and visual beat-level perception in children, and formal musical training was related to enhanced auditory and visual measure-level perception in adults. These findings suggest that modality differences exist early in development and persist through adulthood, and experience improves beat and meter perception in both modalities.

It is possible that the auditory advantage for beat perception arises at least in part from inherently greater temporal acuity of the auditory system. The auditory system is more temporally precise than the visual system for both single-interval and multiple-interval (rhythm) timing (Grondin, 1993, 2010; Grondin \& McAuley, 2009; Merchant et al., 2008; Patel et al., 2005; Repp, 2003; Repp \& Penel, 2002). Additionally, the temporal binding window is also wider (more tolerant of asynchrony) for visual information leading auditory information than the reverse across a wide age range (Hillock et al., 2011; Hillock-Dunn \& Wallace, 2012; Lewkowicz \& Flom 2014; Stevenson, Altieri, et al., 2010; Stevenson, Zemtsov, et al., 2012). Thus, both the better temporal precision of the auditory system and the greater tolerance for visual (metronomes) leading auditory (musical beat) information could have contributed to the auditory advantage for beat perception we observed in children and adults. Future research could probe the contribution of sensory acuity and the temporal binding window to the auditory advantage in cross-modal meter perception by manipulating whether visual or auditory information leads in beat-mismatching conditions, and by how much. However, it is unlikely that the auditory advantage arises from modality-specific beat perception mechanisms: beat perception in visual and auditory tasks is associated with highly similar and overlapping patterns of neural activation (Grahn et al., 2011; Hove et al., 2013; Marchant \& Driver, 2013; Pasinski et al., 2016; Schubotz et al., 2000). This could arise from a shared mechanism for beat perception in non-sensory areas of the brain, or by visual rhythms being "recoded" as auditory rhythms in 
the auditory cortex (e.g., Guttman et al., 2005; McAuley \& Henry, 2010). In either case, the greater temporal precision of the auditory system would lead to more accurate determination of the temporal location of events in the metronome or music, at least at the beat level.

While we observed an auditory advantage for beat perception, we did not find an auditory advantage for measure perception. Indeed, slow, continuous visual motion across longer time scales may contribute to the perception of temporal regularities and global tempo stability in music (Colley et al., 2018; MacRitchie et al., 2017). Measure-level information in music occurs at a slower timescale (hundreds-of-milliseconds-to-seconds range) than the beat-level timescale (few hundreds of milliseconds). There is increasing evidence that temporal processing in the tens to hundreds of milliseconds range may occur in sensory-specific areas, while longer timing windows from the hundreds to thousands of milliseconds may occur in a modality-general or sensory-overlapping timing system (such as secondary auditory cortex) with input from sensoryspecific areas (Buhusi \& Meck, 2005; Ivry \& Schlerf, 2008; Karmakar \& Buonomano, 2007; Lewis \& Miall, 2003; Merchant et al., 2008; Nani et al., 2019; Paton \& Buonomano, 2018; Rammsayer \& Pichelmann, 2018; Stauffer et al., 2012; van Wassenhove, 2009). Motor areas of the brain, including the supplementary motor area (SMA), pre-SMA, premotor cortex, and basal ganglia, are consistently implicated in beat perception and sensorimotor synchronization tasks (Chapin et al., 2010; Chen et al., 2008; Grahn \& Brett, 2007; Grahn \& Rowe, 2009; Hove et al., 2013) for both auditory and visual stimuli. This type of large, distributed network of brain regions that can maintain activity in the absence of external stimulation may be more suited to maintaining temporal expectancies across longer time periods - such as the supra-second period of measure-level beats - than sensory areas (Buonomano \& Laje, 2010; Kotz et al., 2016; Merchant et al., 2015; Merchant \& Yarrow, 2016; Paton \& Buonomano, 2018; Schwartze \& 
Kotz, 2013). These modality-general networks are likely multipurpose: beat and meter perception shows very similar patterns of neural activation as working memory, for example $(\mathrm{Gu}$ et al., 2015).

The transition from modality-specific to modality-general timing mechanisms is hypothesized to occur somewhere in the range of 100-500ms (Buonomano et al., 2009; Karmarkar \& Buonomano, 2007; Spencer et al., 2009). Thus, the auditory advantage for beat perception could arise from a stronger influence of modality-specific temporal processing at the faster beat timescale, whereas the similar sensitivity to measure-level information across modalities at slower timescales could reflect the stronger influence of modality-general timing mechanisms. Future research, especially using brain-imaging, could systematically compare beat and measure perception across modalities using music with very fast (e.g., $300 \mathrm{~ms}$ ) and very slow (e.g., $1000 \mathrm{~ms}$ ) beat periods while examining neural activity in modality-specific sensory areas and in broader networks that include the motor system. In such studies, one may expect to see a stronger auditory advantage in perception for the fast beat along with greater activation in sensory areas, and either a small or no auditory advantage for perception of the slow beat along with greater activation of modality-general or motor networks. Alternatively, if visual beat perception relies on or benefits from auditory "recoding" at faster beat periods (Guttman et al., 2005; McAuley \& Henry, 2010), one might observe high levels of activation in primary and secondary auditory areas for both visual and auditory beat perception at faster, but not slower, beat periods.

We found that formal training was generally related to enhanced perception in both modalities, although the effects were smaller than anticipated and somewhat variable across experiments. Perhaps surprisingly, we did not find that training was associated with a greater 
auditory advantage. Music training related to stronger perception of measure in both modalities in adults (Experiments $1 \& 2$ ), even when controlling for performance on an unrelated musical rhythm perception task (Experiment 2). In children, music training related to enhanced beat perception (Experiment 3). Musicians consistently demonstrate greater sensitivity to the beat and outperform non-musicians on auditory tests of rhythm and beat perception (Grahn \& Rowe, 2009; Karpati et al., 2016; Matthews et al., 2016). Our results demonstrate this enhanced sensitivity related to formal training extends to the perception of slower beat levels in both the auditory and visual modalities. Dance training generally related to poorer measure-level perception as compared to non-dancers, whether across both modalities (in Experiment 1) or specifically in visual metronomes (Experiment 2). However, we interpret our results with caution as the overall level of training - especially dance training - was low across all experiments. Future experiments examining the effect of training would benefit from recruiting groups with greater amounts of formal training to obtain a clearer picture of the relationships between consistent dance (and music) training and perception.

Training and experience could lead to improvements in beat and meter perception through both explicit and implicit means. Greater explicit musical knowledge (e.g., concepts of beat, measure, and meter) may help individuals identify musical structure and generalize information across modalities. Greater implicit experience with culturally-regular rhythmic patterns could also lead to stronger meter perception. Individuals perceive beats where a strong expectancy arises from repeatedly presented patterns in the rhythm (Desain \& Honing, 2003; Hannon, Snyder, et al., 2004; Jones \& Pfordresher, 1997; London et al., 2017; Palmer \& Krumhansl, 1990; van der Weij et al., 2017), so musicians' and dancers' increased experience with rhythms could lead to a stronger perception of the meter than casual listeners. However, 
both implicit and explicit improvement likely require focused, engaged experience: future research may benefit from a more fine-grained and standardized measure of training and experience than using years of training as a proxy measurement (e.g., PROMS [Law \& Zentner, 2012], GOLD-MSI [Müllensiefen et al., 2014], GOLD-DSI [Rose et al., 2020]).

We observed an auditory advantage for beat perception in children as young as five years old. Additionally, we found that the auditory advantage increased with age and experience. Part of the changes in the auditory advantage may be due to sensory development and maturation. For example, when perceiving speech in noisy or multi-talker situations, younger children require higher initial signal-to-noise ratios (SNR) to achieve comparable performance to older children and adults, but once performance levels are equalized, decreasing the SNR by the same amount leads decreases performance equally across all ages (Fallon et al., 2000; Fallon et al., 2002). This suggests the limiting factor in this case is auditory sensory maturation, rather than experience and semantic knowledge. Lifespan studies of multisensory integration indicate that younger children consistently integrate auditory and visual information even when the visual information is irrelevant to the task (e.g., in sound localization judgments), as compared to older children who rely more on the more task-appropriate sense (Parker \& Robinson, 2018, Petrini et al., 2015). As children's auditory capabilities improve with age, and they gradually rely less on visual and more on auditory information for temporal judgments, the auditory advantage for beat perception might therefore increase.

Our findings of a robust auditory advantage present in early childhood that increases with age could also indicate the auditory advantage is partially influenced by greater experience with auditory rhythms. However, overall sensitivity to beat was strongly moderated by age, regardless of modality. It is possible that the improvements in beat perception in both modalities with 
increasing age result from the refinement of mechanisms and strategies acquired during auditory experiences, which are then applied to visual beat perception (Grahn et al., 2011; Guttman, et al., 2005; McAuley \& Henry, 2010). Future studies are needed that include infants and pre-school aged children along with older adolescents and older adults to measure the auditory advantage of beat and meter perception more thoroughly across development. We believe our task is wellsuited for studying this throughout childhood and into older adulthood, but care needs to be taken to create and use a task that facilitates fair comparison of our results and the same construct in younger children (Creel \& Quam, 2015).

While children became increasingly sensitive to the beat with age, they did not demonstrate sensitivity to measure-level matching in either modality as a group, unlike adults. There may be two factors at play here: first, the slower tempo of the measure level (hundreds to thousands of milliseconds) may be above children's optimal rate of event tracking. Children's preferences and spontaneous production of rhythmic patterns is faster than adults, and slows with age from an average of $\sim 300 \mathrm{~ms}$ at $4-5$ years to an average of $\sim 600 \mathrm{~ms}$ in adulthood (Drake, Jones, et al., 2006; McAuley et al., 2006). This may reflect inherent limitations in the temporal frequencies that children can track, leaving them unable to perceive a consistent measure-level beat across a longer, supra-second timescale. Beat and meter perception in adulthood presumably relies on a well-developed motor system (Chen et al., 2008; Fujioka et al., 2015; Grahn \& Brett, 2007; Marchant \& Driver, 2013; Sakai et al., 1999; Schubotz et al., 2000; Snyder \& Large, 2005). By contrast, it is possible that children's immature motor and sensory systems dynamically interact and limit each other, given that children's synchronization with metronomes and music improves gradually with age (Braun Janzen et al., 2014; Drake, Jones, et al., 2000; Eerola et al., 2006; Fujii et al., 2014; Ilari, 2015; Kirschner \& Ilari, 2014; Kirschner \& 
Tomasello, 2009; McAuley et al., 2006; Thompson et al., 2005; Woodruff Carr et al., 2016;

Zentner \& Eerola, 2010), as does their perception of the beat (Einarson \& Trainor, 2016; Nave-

Blodgett et al., 2021; Puyjarinet et al., 2017).

Second, perception of slower metrical levels may also depend on the accumulation of acquired knowledge and experience with the music in one's culture. Our finding that music training enhances sensitivity to measure-level perception in adults is consistent with this idea. Just as native speakers are increasingly able to attend to slower levels of linguistic structure with experience and age (Ding et al., 2017), older children and adults become more able to attend to slower temporal structures in music (Drake, Jones, et al., 2000). The gradual acquisition of experience is also hypothesized to underlie the protracted development of phonemic categorization in speech that extends well into adolescence (McMurray et al., 2018), along with the improvements in face recognition ability that continue throughout development and do not peak until approximately age 30 (Germine et al., 2011; Susilo et al., 2013). Overall, the development of meter perception during childhood may require motor and sensory maturation that occurs in tandem with culture-specific listening experience.

In the current study, we used complex, naturalistic music to evoke the perception of meter in our participants, and only observed an auditory advantage for beat, but not measure perception. Would our results have differed if we had used a complex visual rhythm (e.g., silent dancing) against which the auditory and visual metronomes were judged? Currently, little is known about whether individuals perceive beat and measure in visual rhythms. When individuals tap to the beat of an auditory rhythm, they tap slower if there are additional metrical levels present (Repp, 2008). When asked to tap to the beat in silent point-light "dancing" figures, adults tapped at a slower rate than the actual tempo of the movements when they were asked to tap to a 
slower movement cycle in the dance (e.g., torso versus limb movements; Su, 2016a, 2016b; Su \& López, 2016). This could be evidence that the participants perceived multiple levels of meter in the visual rhythms, but it is not conclusive. Future research needs to first establish if individuals perceive multiple beat levels in response to a complex visual rhythm (e.g., point-light figures, dance videos, etc.), or if only auditory rhythms evoke perception of more than a single beat level. Comparisons between modalities could then better determine the neural mechanisms that underpin beat and measure perception and establish the scope of the auditory advantage for rhythm perception.

In summary, we provide evidence that an auditory advantage exists for beat-level perception, but not measure-level perception. This auditory advantage is present early in development, suggesting a contribution from sensory-level differences, and increases with age, suggesting a contribution from increasing experience with auditory rhythms. More music training was associated with greater sensitivity to beat (in children) and measure (in adults) in both audition and vision. This suggests that expertise plays a role in directing attention to the temporal hierarchies in rhythms and enhancing meter perception across modalities. The auditory advantage for beat perception may arise in part from inherent sensory differences, while acquired experience with the rhythmic regularities of our environment may also influence the size of the auditory advantage and the development of meter perception in audition and vision. 


\section{References}

Alais, D., \& Burr, D. (2004). The ventriloquist effect results from near-optimal bimodal integration. Current Biology, 14, 257-262.

Audacity Team (2021). Audacity®: Free Audio Editor and Recorder (version 2.1.2) [Computer software]. Retrieved from https://www.audacityteam.org

Baayen, R. H., Davidson, D. J., \& Bates, D. M. (2008). Mixed-effects modeling with crossed random effects for subjects and items. Journal of Memory and Language, 59, 390-412.

Bahrick, L. E., Flom, R., \& Lickliter, R. (2002). Intersensory redundancy facilitates discrimination of tempo in 3-month-old infants. Developmental Psychobiology, 41, 352363.

Bahrick, L. E., \& Lickliter, R. (2004). Infants' perception of rhythm and tempo in unimodal and multimodal stimulation: A developmental test of the intersensory redundancy hypothesis. Cognitive, Affective, \& Behavioral Neuroscience, 4(2), 137-147.

Baruch, C., \& Drake, C. (1997). Tempo discrimination in infants. Infant Behavior and Development, 20(4), 573-577.

Bates, D., Maechler, M., Bolker, B., \& Walker, S. (2015). Fitting Linear Mixed-Effects Models Using lme4. Journal of Statistical Software, 67(1), 1-48. DOI:10.18637/jss.v067.i01.

Beier, E. J., \& Ferreira, F. (2018). The temporal prediction of stress in speech and its relation to musical beat perception. Frontiers in Psychology, 9, 431.

Bidelman, G. M. (2016). Musicians have enhanced audiovisual multisensory binding: experience-dependent effects in the double-flash illusion. Experimental Brain Research, 234(10), 3037-3047. 
Brandon, M., \& Saffran, J. R. (2011). Apparent motion enhances visual rhythm discrimination in infancy. Attention, Perception, \& Psychophysics, 73, 1016-1020.

Braun Janzen, T., Thompson, W. F., \& Ranvaud, R. (2014). A developmental study of the effect of music training on timed movements. Frontiers in Human Neuroscience, 8, 801.

Buhusi, C. V., \& Meck, W. H. (2005). What makes us tick? Functional and neural mechanisms of interval timing. Nature Reviews Neuroscience, 6(10), 755-765.

Buonomano, D. V., Bramen, J., \& Khodadadifar, M. (2009). Influence of the interstimulus interval on temporal processing and learning: testing the state-dependent network model. Philosophical Transactions of the Royal Society B, 364, 1865-1873.

Buonomano, D. V., \& Laje, R. (2010). Population clocks: Motor timing with neural dynamics. Trends in Cognitive Science, 14(12), 520-527.

Calvo-Merino, B., Ehrenberg, S., Leung, D., \& Haggard, P. (2010). Experts see it all: configural effects in action observation. Psychological Research, 74(4), 400-406.

Celma-Miralles, A., de Menezes, R. F., \& Toro, J. M. (2016). Look at the beat, feel the meter: Top-down effects of meter induction on auditory and visual modalities. Frontiers in Human Neuroscience, 10, 108.

Celma-Miralles, A., \& Toro, J. M. (2019). Ternary meter from spatial sounds: Differences in neural entrainment between musicians and non-musicians. Brain and Cognition, 136, 103594.

Chapin, H. L., Zanto, T., Jantzen, K. J., Kelso, S. J. A., Steinberg, F., \& Large, E. W. (2010). Neural responses to complex auditory rhythms: The role of attending. Frontiers in Psychology, 1, 224. 
Chen, J. L., Penhune, V. B., \& Zatorre, R. J. (2008). Listening to musical rhythms recruits motor regions of the brain. Cerebral Cortex, 18, 2844-2854.

Cheour-Luhtanen, M., Alho, K., Saino, K., Rinne, T., Reinikainen, K., Pohjavuori, M., Renlund, M., Aaltonen, O., Eerola, O., \& Näätänen, R. (1996). The ontogenetically earliest discriminative response of the human brain. Psychophysiology, 33, 478-481.

Colley, I. D., Varlet, M., MacRitchie, J., \& Keller, P. E. (2018). The influence of visual cues on temporal anticipation and movement synchronization with musical sequences. Acta Psychologica, 191, 190-200.

Cousineau, D. (2005). Confidence intervals in within-subject designs: A simpler solution to Loftus and Masson's method. Tutorials in Quantitative Methods for Psychology, 1(1), $42-45$.

Davies, M. P., \& Plumbley, M. D. (2007). Context-dependent beat tracking of musical audio. IEEE Transactions on Audio, Speech, and Language Processing, 15(3), 1009-1020.

Dawson, J.F., \& Richter, A.W. (2006). Probing three-way interactions in moderate multiple regression: Development and application of a slope difference test. Journal of Applied Psychology, 91, 917-926.

Demany, L., McKenzie, B., \& Vurpillot, E. (1977). Rhythm perception in early infancy. Nature, 266(5604), 718.

Desain, P., \& Honing, H. (2003). The formation of rhythmic categories and metric priming. Perception, 32(3), 341-365.

Ding, N., Melloni, L., Zhang, H., Tian, X., \& Poeppel, D. (2016). Cortical tracking of hierarchical linguistic structures in connected speech. Nature Neuroscience, 19(1), 158164. 
Ding, N., Patel, A. D., Chen, L., Butler, H., Luo, C., \& Poeppel, D. (2017). Temporal modulations in speech and music. Neuroscience and Biobehavioral Reviews, 81, 181187.

Drake, C. (1993). Reproduction of musical rhythms by children, adult musicians, and adult nonmusicians. Perception \& Psychophysics, 53(1), 25-33.

Drake, C., Jones, M. R., \& Baruch, C. (2000). The development of rhythmic attending in auditory sequences: attunement, referent period, focal attending. Cognition, 77, 251-288.

Drake, C., Penel, A., \& Bigand, E. (2000). Tapping in time with mechanically and expressively performed music. Music Perception, 18(1), 1-23.

Essens, P. J., \& Povel, D. J. (1985). Metrical and nonmetrical representations of temporal patterns. Perception \& Psychophysics, 37(1), 1-7.

Fallon, M., Trehub, S. E., \& Schneider, B. A. (2000). Children's perception of speech in multitalker babble. Journal of the Acoustical Society of America, 108(6), 3023-3029.

Fallon, M., Trehub, S. E., \& Schneider, B. A. (2002). Children's use of semantic cues in degraded listening environments. Journal of the Acoustic Society of America, 111(5), 2242-2249.

Fraisse, P. (1982). Rhythm and tempo. In Deutsch, D. (Ed) The Psychology of Music (1 ${ }^{\text {st }}$ edition), 149-180.

Fritz, C. O., Morris, P. E., \& Richler, J. J. (2012). Effect size estimates: Current use, calculations, and interpretation. Journal of Experimental Psychology: General, 141(1), 2-18.

Fujii, S., Watanabe, H., Oohashi, H., Hirashima, M., Nozaki, D., \& Taga, G. (2014). Precursors of dancing and singing to music in three- to four-months-old infants. PloS One, 9(5), e97680. 
Fujioka, T., Ross, B., \& Trainor, L. J. (2015). Beta-band oscillations represent auditory beat and its metrical hierarchy in perception and imagery. The Journal of Neuroscience, 35(45), 15187-15198.

Gault, R. H., \& Goodfellow, L. D. (1938). An empirical comparison of audition, vision, and touch in the discrimination of temporal patterns and the ability to reproduce them. The Journal of General Psychology, 18(1), 41-47.

Geiser, E., Sandmann, P., Jäncke, L., \& Meyer, M. (2010). Refinement of metre perception training increases hierarchical metre processing. European Journal of Neuroscience, 32, 1979-1985.

Germine, L. T., Duchaine, B., \& Nakayama, K. (2011). Where cognitive development and aging meet: Face learning ability peaks after age 30. Cognition, 118, 201-210.

Gordon, E. E. (1986). Advanced Measures of music audiation. Chicago, IL: G.I.A. Publications.

Grahn, J. A. (2012). See what I hear? Beat perception in auditory and visual rhythms. Experimental Brain Research, 220, 51-61.

Grahn, J. A., \& Brett, M. (2007). Rhythm and beat perception in motor areas of the brain. Journal of Cognitive Neuroscience, 19(5), 893-906.

Grahn, J. A., Henry, M. J., \& McAuley, J. D. (2011). FMRI investigation of cross-modal interactions in beat perception: Audition primes vision, but not vice-versa. NeuroImage, $54,1231-1243$.

Grahn, J. A., \& Rowe, J. B. (2009). Feeling the beat: Premotor and striatal interactions in musicians and non-musicians during beat perception. The Journal of Neuroscience, 29(23), 7540-7548. 
Grahn, J. A., \& Schuit, D. (2012). Individual differences in rhythmic ability: Behavioral and neuroimaging investigations. Psychomusicology: Music, Mind, \& Brain, 22(2), 105-121.

Grondin, S. (1993). Duration discrimination of empty and filled intervals marked by auditory and visual signals. Perception \& Psychophysics, 54, 383-394.

Grondin, S. (2010). Timing and time perception: A review of recent behavioral and neuroscience findings and theoretical directions. Attention, Perception, \& Psychophysics, 72(3), 561582.

Grondin, S., \& McAuley, J. D. (2009). Duration discrimination in crossmodal sequences. Perception, 38, 1542-1559.

Gu, B.-M., van Rijn, H., \& Meck, W. H. (2015). Oscillatory multiplexing of neural population codes for interval timing and working memory. Neuroscience and Biobehavioral Reviews, 48, 160-185.

Guttman, S. E., Gilroy, L. A., \& Blake, R. (2005). Hearing what the eyes see: auditory encoding of visual temporal sequences. Psychological Science, 16(3), 228-235.

Handel, S., \& Oshinsky, J. S. (1981). The meter of syncopated auditory polyrhythms. Perception \& Psychophysics, 30(1), 1-9.

Hannon, E. E., \& Johnson, S. P. (2005). Infants use meter to categorize rhythms and melodies: Implications for musical structure learning. Cognitive Psychology, 50, 354-377.

Hannon, E. E., Nave-Blodgett, J. E., \& Nave, K. M. (2018). The developmental origins of the perception and production of musical rhythm. Child Development Perspectives, 12, 194198. doi:10.1111/cdep. 12285

Hannon, E. E., Schachner, A., \& Nave-Blodgett, J. E. (2017). Babies know bad dancing when they see it: Older but not younger infants discriminate between synchronous and 
asynchronous audiovisual musical displays. Journal of Experimental Child Psychology, 159, 159-174.

Hannon, E. E., Soley, G., \& Levine, R. S. (2011). Constraints on infants' musical rhythm perception: Effects of interval ratio complexity and enculturation. Developmental Science, 14(4), 865-872.

Hannon, E. E., Soley, G., \& Ullal, S. (2012). Familiarity overrides complexity in rhythm perception: A cross-cultural comparison of American and Turkish listeners. Journal of Experimental Psychology: Human Perception and Performance, 38(3), 543-548.

Hannon, E. E., Snyder, J. S., Eerola, T., \& Krumhansl, C. L. (2004). The role of melodic and temporal cues in perceiving musical meter. Journal of Experimental Psychology: Human Perception and Performance, 30(5), 956-974.

Hannon, E. E., \& Trehub, S. E. (2005). Metrical categories in infancy and adulthood. Psychological Science, 16(1), 48-55.

Hannon, E. E., Vanden Bosch der Nederlanden, C. M., \& Tichko, P. (2012). Effects of perceptual experience on children's and adults' perception of unfamiliar rhythms. Annals of the New York Academy of Sciences, 1252, 92-99.

Hartmann, W. M., \& Johnson, D. (1991). Stream segregation and peripheral channeling. Music Perception, 9(2), 155-183.

Hillock, A. R., Powers, A. R., \& Wallace, M. T. (2011). Binding of sights and sounds: Agerelated changes in multisensory temporal processing. Neuropsychologia, 49, 461-467.

Hillock-Dunn, A., Grantham, D. W., \& Wallace, M. T. (2016). The temporal binding window for audiovisual speech: Children are like little adults. Neuropsychologia, 88, 74-82. 
Hillock-Dunn, A., \& Wallace, M. T. (2012). Developmental changes in the multisensory temporal binding window persist into adolescence. Developmental Science, 15(5), 688696.

Hove, M. J., Fairhurst, M. T., Kotz, S. A., \& Keller, P. E. (2013). Synchronizing with auditory and visual rhythms: An fMRI assessment of modality differences and modality appropriateness. Neuroimage, 67, 313-321.

Hove, M. J., Iversen, J. R., Zhang, A., \& Repp, B. H. (2013). Synchronization with competing visual and auditory rhythms: bouncing ball meets metronome. Psychological Research, 77, 388-399.

Hove, M. J., Spivey, M. J., \& Krumhansl, C. L. (2010). Compatibility of motion facilitates visuomotor synchronization. Journal of Experimental Psychology: Human Perception and Performance, 36(6), 1525-1534.

Hox, J. J. (1995). Applied multilevel analysis. TT-Publikaties.

Hox, J. J. (2010). Multilevel analysis techniques and applications. ( $2^{\text {nd }}$ Ed.) Routledge.

Huang, J., Gamble, D., Sarnlertsophon, K., Wang, X., \& Hsiao, S. (2012). Feeling music: Integration of Auditory and Tactile Inputs in Musical Meter Perception. PLoS One, 7(10), e48496.

Huff, J. (1972). Auditory and visual perception of rhythm by performers skilled in selected motor activities. Research Quarterly: American Asssociation for Health, Physical Education, and Recreation, 43(2), 197-207.

Ilari, B. (2015). Rhythmic engagement with music in early childhood: A replication and extension. Journal of Research in Music Education, 62, 332-343. 
Innes-Brown, H., Barutchu, A., Shivdasani, M. N., Crewther, D. P., Grayden, D. B., \& Paolini, A. G. (2011). Susceptibility to the flash-beep illusion is increased in children compared to adults. Developmental Science, 14(5), 1089-1099.

Iversen, J. R., Patel, A. D., Nicodemus, B., \& Emmorey, K. (2015). Synchronization to auditory and visual rhythms in hearing and deaf individuals. Cognition, 134, 232-244.

Ivry, R. B., \& Schlerf, J. E. (2008). Dedicated and intrinsic models of time perception. Trends in Cognitive Sciences, 12(7), 273-280.

Jin, X., Wang, B., Lv, Y., Lu, Y., Chen, J., \& Zhou, C. (2019). Does dance training influence beat sensorimotor synchronization? Differences in finger-tapping sensoritmoro synchronization between competitive ballroom dancers and nondancers. Experimental Brain Research, 237, 743-753.

Jones, M. R. (1976). Time, our lost dimension: Toward a new theory of perception, attention, and memory. Psychological Review, 83(5), 323-355.

Jones, M. R., \& Boltz, M. (1989). Dynamic attending and responses to time. Psychological Review, 96(3), 459-491.

Jones, M. R., \& Pfordresher, P. Q. (1997). Tracking musical patterns using joint accent structure. Canadian Journal of Experimental Psychology, 51, 271-291.

Jusczyk, P.W., Friederici, A. D., Wessels, J. M. I., Svenkerud, V. Y., \& Jusczyk, A. M. (1993). Infants' sensitivity to the sound patterns of native language words. Journal of Memory and Language, 32, 402-420.

Karmakar, U. R., \& Buonomano, D. V. (2007). Timing in the absence of clocks: Encoding time in neural network states. Neuron, 53, 427-238. 
Karpati, F. J., Giacosa, C., Foster, N. E. V., Penhune, V. B., \& Hyde, K. L. (2016). Sensorimotor integration is enhanced in dancers and musicians. Experimental Brain Research, 234(3), 893-903.

Kello, C. T., Dalla Bella, S., Médé, B., \& Balasubramaniam, R. (2017). Hierarchical temporal structure in music, speech, and animal vocalizations: Jazz is like a conversation, humpbacks sing like hermit thrushes. Journal of the Royal Society Interface, 14, 20170231.

Kirschner, S. \& Tomasello, M. (2009). Joint drumming: Social context facilitates synchronization in preschool children. Journal of Experimental Child Psychology, 102(3), 299-314.

Kirschner, S., \& Ilari, B. (2014). Joint drumming in Brazilian and German preschool children: Cultural differences in rhythmic entrainment, but no prosocial effects. Journal of CrossCultural Psychology, 45(1), 137-166.

Kosonen, K., \& Raisamo, R. (2006). Rhythm perception through different modalities. In Proc. EuroHaptics (pp. 365-370).

Kurgansky, A. V., \& Shupikova, E. S. (2011). Visuomotor synchronization in adults and sevento eight-year-old children. Human Physiology, 37(5), 526-536.

Kuznetsova, A., Brockhoff, P. B., \& Christensen, R. H. B. (2017). lmerTest package: Tests in linear mixed effects models. Journal of Statistical Software, 82(13), 1-26. https://doi.org/10.18637/JSS.V082.I13

Large, E. W., \& Jones, M. R. (1999). The dynamics of attending: How people track time-varying events. Psychological Review, 106(1), 119-159. 
Law, L. N. C., \& Zentner, M. (2012). Assessing musical abilities objectively: Construction and validation of the Profile of Music Perception Skills, PLoS One, 7(12), e52508.

Lehrdahl, F., \& Jackendoff, R. (1985). A generative theory of tonal music. MIT Press.

Lester, J. (1986). The rhythms of tonal music. Southern Illinois University Press.

Lewis, P. A., \& Miall, R. C. (2003). Brain activation patterns during measurement of sub- and supra-second intervals. Neuropsychologia, 41, 1583-1592.

Lewkowicz, D. J., \& Flom, R. (2014). The audiovisual temporal binding window narrows in early childhood. Child Development, 85(2), 685-694.

London, J. (2002). Cognitive constraints on metric systems: Some observations and hypotheses. Music Perception, 19(4), 529-550.

London, J. (2012). Hearing in time. Oxford University Press.

London, J., Polak, R., \& Jacoby, N. (2017). Rhythm histograms and musical meter: a corpus study of Malian percussion music. Psychonomic Bulletin \& Review, 24(2), 474-480.

MacRitchie, J., Varlet, M., \& Keller, P. E. (2017). Embodied expression through entrainment and co-representation in musical ensemble performance. In The Routledge companion to embodied music interaction (pp. 150-159). Routledge.

Manning, F. C., \& Schutz, M. (2016). Trained to keep a beat: movement-related enhancements to timing perception in percussionists and non-percussionists. Psychological Research, $80(4), 532-542$.

Marchant, J. I., \& Driver, J. (2013). Visual and audiovisual effects of isochronous timing on visual perception and brain activity. Cerebral Cortex, 23, 1290-1298. 
Matthews, T. E., Thibodeau, J. N. L., Gunther, B. P., \& Penhune, V. B. (2016). The impact of instrument-specific musical training on rhythm perception and production. Frontiers in Psychology, 7, 69.

McAuley, J. D., \& Henry, M. J. (2010). Modality effects in rhythm processing: Auditory encoding of visual rhythms is neither obligatory or automatic. Attention, Perception, \& Psychophysics, 72(5), 1377-1389.

McAuley, J. D., Jones, M. R., Holub, S., \& Johnson, H. M. (2006). The time of our lives: Life span development of timing and event tracking. Journal of Experimental Psychology: General, 135(3), 348-367.

McMurray, B., Danelz, A., Rigler, H., \& Seedorff, M. (2018). Speech categorization develops slowly through adolescence. Developmental Psychology, 54(8), 1472-1491.

Merchant, H., Grahn, J., Trainor, L., Rohrmeier, M., \& Fitch, W. T. (2015). Finding the beat: A neural perspective across humans and non-human primates. Philosophical Transactions of the Royal Society B, 37,

Merchant, H., \& Yarrow, K. (2016). How the motor system both encodes and influences our sense of time. Current Opinion in Behavioral Sciences, 8, 22-27.

Merchant, H., Zarco, W., \& Prado, L. (2008). Do we have a common mechanism for measuring time in the hundreds of millisecond range? Evidence from multiple-interval timing tasks. Journal of Neurophysiology, 99, 939-949.

Miura, A., Kudo, K., Ohtsuki, T., \& Kanehisa, H. (2016). Coordination modes in sensorimotor synchronization of whole-body movement: A study of street dancers and non-dancers. Human Movement Science, 30, 1260-1271. 
Mu, Y., Huang, Y., Ji, C., Gu, L., \& Wu, X. (2017). Auditory over visual advantage of sensorimotor synchronization in 6- to 7-year old children but not in 12-to-15-year-old children and adults. Journal of Experimental Psychology: Human Perception and Performance, 44(5), 818-826.

Müllensiefen, D., Gingras, B., Musil, J., \& Stewart, L. (2014). Measuring the facets of musicality: The Goldsmiths Musical Sophistication Index (Gold-MSI). Personality and Individual Differences, 60, S35.

Nani, A., Manuello, J., Liloia, D., Duca, S., Costa, T., \& Cauda, F. (2019). The neural correlates of time: A meta-analysis of neuroimaging studies. Journal of Cognitive Neuroscience, 31(12), 1796-1826.

Nava, E., \& Pavani, F. (2013). Changes in sensory dominance during childhood: Converging evidence from the Colavita effect and the sound-induced flash illusion. Child Development, 84(2), 604-616.

Nave-Blodgett, J. E., Hannon, E. E., \& Snyder, J. S. (2020, July 14). Auditory superiority for perceiving the beat level but not measure level in music. OSF Project: https://osf.io/xgsvk/

Nave-Blodgett, J. E., Snyder, J. S., \& Hannon, E. E. (2021). Hierarchical beat perception develops throughout childhood and adolescence and is enhanced in those with musical training. Journal of Experimental Psychology: General, 150(2), 314-339. https://doi.apa.org/doi/10.1037/xge0000903

Naveda, L., \& Leman, M. (2010). The spatiotemporal representation of dance and music gestures using topological gesture analysis (TGA). Music Perception, 28(1), 93-111. 
Naveda, L., Martinez, I. C., Damesón, J., Ghiena, A. P., Hererra, R., \& Ordás, M. O. (2015). Cross-cultural comparisons of unconstrained body responses to Argentinian and AfroBrazilian music. In International Symposium on Computer Music Multidisciplinary Research, 464-482.

Nazzi, T., Bertoncini, J., \& Mehler, J. (1998). Language discrimination by newborns: Toward an understanding of the role of rhythm. Journal of Experimental Psychology: Human Perception and Performance, 24(3), 756-766.

Palmer, C., \& Krumhansl, C. L. (1990). Mental representations for musical meter. Journal of Experimental Psychology: Human Perception and Performance, 16(4), 728-741.

Palmer, S. E., \& Peterson, J. (2016). Hierarchical structure of musical and visual meter in crossmodal "fit" judgments. Proceedings of the $14^{\text {th }}$ International Conference on Music Cognition and Perception, San Francisco, CA.

Parker, J. L., \& Robinson, C. W. (2018). Changes in multisensory integration across the lifespan. Psychology and Aging, 33(3), 545-558.

Parncutt, R. (1994). A perceptual model of pulse salience and metrical accent in musical rhythms. Music Perception, 11(4), 409-464.

Pasinski, A. C., McAuley, J. D., \& Snyder, J. S. (2016). How modality-specific is processing of auditory and visual rhythms? Psychophysiology, 53, 198-208.

Patel, A. D., Iversen, J. R., Chen, Y., \& Repp, B. H. (2005). The influence of metricality and modality on synchronization with a beat. Experimental Brain Research, 163, 226-238.

Paton, J. J., \& Buonomano, D. V. (2018). The neural basis of timing: Distributed mechanisms for diverse functions. Neuron, 98, 687-705. 
Petrini, K., Jones, P. R., Smith, L., \& Nardini, M. (2015). Hearing where the eyes see: Children use an irrelevant visual cue when localizing sounds. Child Development, 86(5), 14491457.

Povel, D. J. (1981). Internal representation of simple temporal patterns. Journal of Experimental Psychology: Human Perception and Performance, 7(1), 3.

Povel, D. J., \& Essens, P. (1985). Perception of temporal patterns. Music Perception: An Interdisciplinary Journal, 2(4), 411-440.

Preacher, K. J., Curran, P. J., \& Bauer, D. J. (2006). Computational tools for probing interactions in multiple linear regression, multilevel modeling, and latent curve analysis. Journal of Educational and Behavioral Statistics, 31(4), 437-448.

Provasi, J., \& Bobin-Bègue, A. (2003). Spontaneous motor tempo and rhythmical synchronisation in 21/2- and 4-year-old children. International Journal of Behavioral Development, 27(3), 220-231.

Puyjarinet, F., Bégel, V., Lopez, R., Dellacherie, D., \& Dalla Bella, S. (2017). Children and adults with Attention-Deficit/Hyperactivity Disorder cannot move to the beat. Scientific Reports, 7(1), 11550.

R Core Team (2020). R: A language and environment for statistical computing. R Foundation for Statistical Computing, Vienna, Austria. URL https://www.R-project.org.

Rammsayer, T., \& Altenmüller, E. (2006). Temporal information processing in musicians and nonmusicians. Music Perception, 24(1), 37-48.

Rammsayer, T., Buttkus, F., \& Altenmüller, E. (2012). Musicians do better than nonmusicians in both auditory and visual timing tasks. Music Perception, 30(1), 85-96. 
Rammsayer, T., \& Pichelmann, S. (2018). Visual-auditory differences in duration discrimination depend on modality-specific, sensory-automatic temporal processing: Converging evidence for the validity of the sensory-automatic timing hypothesis. Quarterly Journal of Experimental Psychology, 71(11), 2364-2377.

Raudenbusch, S. W., \& Bryk, A. S. (2002). Hierarchical linear models: Applications and data analysis methods. (2nd ed.) Sage.

Raudenbush, S. W., \& Liu, X. (2000). Statistical Power and Optimal Design for Multisite Randomized Trials. Psychological Methods, 5(2), 199-213.

Repp, B. H. (2003). Rate limits in sensorimotor synchronization with auditory and visual sequences: The synchronization threshold and the benefits and costs of interval subdivision. Journal of Motor Behavior, 35(4), 355-370.

Repp, B. H. (2008). Metrical subdivision results in subjective slowing of the beat. Music Perception, 26(1), 19-39.

Repp, B. H., \& Penel, A. (2002). Auditory dominance in temporal processing: New evidence from synchronization with simultaneous visual and auditory sequences. Journal of Experimental Psychology: Human Perception and Performance, 28(5), 1085-1099.

Repp, B. H., \& Penel, A. (2004). Rhythmic movement is attracted more strongly to auditory than to visual rhythms. Psychological Research, 68, 252-270.

Repp, B. H., \& Su, Y.-H. (2013). Sensorimotor synchronization: A review of recent research (2006-2012). Psychonomic Bulletin Review, 20, 403-452.

Riecke, L., Formisano, E., Sorger, B., Başkent, D., \& Gaudrain, E. (2018). Neural entrainment to speech modulates speech intelligibility. Current Biology, 28, 161-169. 
Rose, D., Müllensiefen, D., Lovatt, P., \& Orgs, G. (2020). The Goldsmiths Dance Sophistication Index (Gold-DSI): A psychometric tool to assess individual differences in dance experience. Psychology of Aesthetics, Creativity, and the Arts. Advance online publication.

Rosenbusch, M. H., \& Gardner, D. B. (1968). Reproduction of visual and auditory rhythm patterns by children. Perceptual and Motor Skills, 26, 1271-1276.

Rubin, D. B. (1987). Multiple imputation for nonresponse in surveys. John Wiley.

Sakai, K., Hikosaka, O., Miyauchi, S., Takino, R., Tamada, T., Iwata, N. K., \& Nielsen, M. (1999). Neural representation of a rhythm depends on its interval ratio. Journal of Neuroscience, 19(22), 10074-10081.

Satterthwaite, F. E. (1946). An approximate distribution of estimates of variance components. Biometrics Bulletin, 2(6), 110-114.

Schellenberg, E. G. (2011). Examining the association between music lessons and intelligence. British Journal of Psychology, 102(3), 283-302. https://doi.org/10.1111/j.20448295.2010.02000.x

Schubotz, R. I., Friederici, A. D., \& von Cramon, D. Y. (2000). Time perception and motor timing: A common cortical and subcortical basis revealed by fMRI. NeuroImage, 11, 112.

Schwartze, M., \& Kotz, S. A. (2013). A dual-pathway neural architecture for specific temporal prediction. Neuroscience and Biobehavioral Reviews, 37, 2587-2596.

Skaansar, J. F., Laeng, B., \& Danielsen, A. (2019). Microtiming and mental effort: Onset asynchronies in musical rhythm modulate pupil size. Music Perception, 37(2), 111-133. 
Snyder, J. S., \& Large, E. W. (2005). Gamma-band activity reflects the metric structure of rhythmic tone sequences. Cognitive Brain Research, 24, 117-126.

Spencer, R. M. C., Karmakar, U., \& Ivry, R. B. (2009). Evaluating dedicated and intrinsic models of temporal encoding by varying context. Philosophical Transactions of the Royal Society B, 364, 1853-1863.

Spybrook, J, Bloom, H., Congdon, R., Hill, C., Martinez, A., \& Raudenbush, S. (2011). Optimal design plus empirical evidence: Documentation for the "Optimal Design" software. Retrieved from http://hlmsoft.net/od/od-manual-20111016-v300.pdf

Stauffer, C. C., Haldemann, J., Troche, S. J., \& Rammsayer, T. H. (2012). Auditory and visual temporal sensitivity: Evidence for a hierarchical structure of modality-specific and modality-independent levels of temporal information processing. Psychological Research, 76, 20-31.

Stevenson, R. A., Altieri, N. A., Kim, S., Pisoni, D. B., James, T. W. (2010). Neural processing of asynchronous audiovisual speech perception. Neuroimage, 49(4), 3308-3318.

Stevenson, R. A., Wilson, M. M., Powers, A. R., \& Wallace, M. T. (2013). The effects of visual training on multisensory temporal processing. Experimental Brain Research, 225, 479489.

Stevenson, R. A., Zemtsov, R. K., \& Wallace, M. T. (2012). Individual differences in the multisensory temporal binding window predict susceptibility to audiovisual illusions. Journal of Experimental Psychology: Human Perception and Performance, 38(6), 15171529.

Su, Y.-H. (2014a). Visual enhancement of auditory beat perception across auditory interference levels. Brain and Cognition, 90, 19-31. 
Su, Y.-H. (2014b). Audiovisual beat induction in complex auditory rhythms: Point-light figure movement as an effective visual beat. Acta Psychologica, 151, 40-50.

Su, Y.-H. (2016a). Visual tuning and metrical perception of realistic point-light dance movements. Scientific Reports, 6, 22774.

Su, Y.-H. (2016b). Sensorimotor synchronization with different metrical levels of point-light dance movements. Frontiers in Human Neuroscience, 10, 186.

Su, Y.-H., \& Salazar-López, E. (2016). Visual timing of structured dance movements resembles auditory rhythm perception. Neural Plasticity, 2016, 1678390. https://doi.org/10.1155/2016/1678390

Susilo, T., Germine, L., \& Duchaine, B. (2013). Face recognition ability matures late: Evidence from individual differences in young adults. Journal of Experimental Psychology: Human Perception and Performance, 39(5), 1212-1217.

Stark, A. M., Davies, M. P., \& Plumbley, M. D. (2009). Real-time beat-synchronous analysis of musical audio. In Proceedings of $12^{\text {th }}$ International Conference on Digital Audio Effects (DAFx), Como, Italy.

Thompson, E. C., White-Schwoch, T., Tierney, A., \& Kraus, N. (2015). Beat synchronization across the lifespan: Intersection of development and musical experience. PLoS One, 10(6), e0128839.

Thorpe, L. A., \& Trehub, S. E. (1989). Duration illusion and auditory grouping in infancy. Developmental Psychology, 25(1), 122.

van der Weij, B., Pearce, M. T., \& Honing, H. (2017). A probabilistic model of meter perception: Simulating enculturation. Frontiers in Psychology, 8, 824. 
van Noorden, L., \& Moelants, D. (1999). Resonance in the perception of musical pulse. Journal of New Music Research, 28(1), 43-66.

van Wassenhove, V. (2009). Minding time in an amodal representational space. Philosophical Transactions of the Royal Society B, 364(1525), 1815-1830.

Washburn, A., DeMarco, M., de Vries, S., Ariyabuddhiphongs, K., Schmidt, R. C., Richardson, M. J., \& Riley, M. A. (2014). Dancers entrain more effectively than non-dancers to another actor's movements. Frontiers in Human Neuroscience, 8, 800.

Wechsler, D., \& Hsiao-pin, C. (2011). WASI-II: Wechsler Abbreviated Scale of Intelligence. Pearson.

Welch, R. B., Duttonhurt, L. D., \& Warren, D. H. (1986). Contributions of audition and vision to temporal rate perception. Perception \& Psychophysics, 39(4), 294-300.

Wickham, H. (2016). ggplot2: Elegant graphics for data analysis. Springer-Verlag.

Woodruff Carr, K., Tierney, A., White-Schwoch, T., \& Kraus, N. (2016). Intertrial auditory neural stability supports beat synchronization in preschoolers. Developmental Cognitive Neuroscience, 17, 76-82.

Zentner, M., \& Eerola, T. (2010). Rhythmic engagement with music in infancy. Proceedings of the National Academy of Sciences, 107, 5768-5773. 